\title{
Longitudinal Multi-omic Phenotyping Reveals Host- microbe Responses to Bariatric Surgery, Glycaemic Control and Obesity
}

Nicholas Penney ( $\square$ n.penney@imperial.ac.uk)

Imperial College London https://orcid.org/0000-0003-0896-6590

Derek Yeung

Imperial College London https://orcid.org/0000-0002-5679-9435

Isabel Garcia-Perez

Imperial College London https://orcid.org/0000-0001-8398-0603

Joram POSMA

Imperial College London https://orcid.org/0000-0002-4971-9003

Aleksandra Kopytek

Imperial College London

Bethany Garratt

Imperial College London

Hutan Ashrafian

Imperial College London https://orcid.org/0000-0003-1668-0672

Gary Frost

Imperial University https://orcid.org/0000-0003-0529-6325

Julian Marchesi

Imperial College London

Sanjay Purkayastha

Imperial College Healthcare NHS Trust

Lesley Hoyles

Nottingham Trent University

Ara Darzi

Imperial College London https://orcid.org/0000-0001-7815-7989

Elaine Holmes

Murdoch University

Article

Keywords: Gut Microbiome, Gut Microbiota, Bariatric Surgery, Diabetes, Obesity, Roux-en-Y Gastric Bypass, Sleeve Gastrectomy, metabolomic 
Posted Date: December 28th, 2021

DOI: https://doi.org/10.21203/rs.3.rs-1146471/v1

License: (c) (i) This work is licensed under a Creative Commons Attribution 4.0 International License. Read Full License

Version of Record: A version of this preprint was published at Communications Medicine on October 7th, 2022. See the published version at https://doi.org/10.1038/s43856-022-00185-6. 


\section{Longitudinal multi-omic phenotyping reveals host-microbe 2 responses to bariatric surgery, glycaemic control and obesity}

3 Nicholas C Penney ${ }^{1,2}$; Derek KT Yeung ${ }^{1,2}$; Isabel Garcia-Perez ${ }^{1}$; Joram M Posma ${ }^{1,3}$; Aleksandra Kopytek ${ }^{1}$; Bethany Garratt ${ }^{2}$; 4 Hutan Ashrafian ${ }^{1,2}$; Gary Frost ${ }^{1}$; Julian R Marchesi ${ }^{1}$; Sanjay Purkayastha ${ }^{2}$; Lesley Hoyles ${ }^{1,4}$; Ara Darzi ${ }^{2,5}$; Elaine Holmes ${ }^{1,6}$

7 Author affiliations:

81 Department of Metabolism, Digestion and Reproduction, Faculty of Medicine, Imperial College London, London, SW7 $92 \mathrm{AZ}, \mathrm{UK}$

102 Department of Surgery and Cancer, Faculty of Medicine, Imperial College London, London, W2 1NY, UK

113 Health Data Research UK, London, NW1 2BE, UK

124 Department of Biosciences, Nottingham Trent University, Nottingham, NG11 8NS, UK

135 Institute of Global Health Innovation, Imperial College London, London, W2 1NY, UK

146 Centre for Computational \& Systems Medicine, Health Futures Institute, Murdoch University, Western Australia, 6150, 15 Australia

19 Corresponding authors:

20 Dr Nicholas C. Penney

21 Department of Surgery and Cancer, Faculty of Medicine, Imperial College London, London, UK

22 n.penney@imperial.ac.uk

$23 \quad 07545461256$

25 Professor Elaine Holmes (Lead Contact):

26 Department of Metabolism, Digestion and Reproduction, Faculty of Medicine, Imperial College London, London, UK

27 elaine.holmes@imperial.ac.uk 


\section{Abstract}

30 Resolution of type-2 diabetes (T2D) is common following bariatric surgery, particularly Roux-en-Y

31 gastric bypass (RYGB). However, the underlying mechanisms have not been fully elucidated. To

32 address this we compared the integrated serum, urine and faecal metabolic profiles of obese

33 participants with and without $T 2 D(n=81, T 2 D=42)$ with participants who underwent RYGB or sleeve

34 gastrectomy (pre and 3-months post-surgery; $n=27$ ), taking diet into account. We co-modelled these

35 data with shotgun metagenomic profiles of the gut microbiota to provide a comprehensive atlas of

36 host-gut microbe responses to bariatric surgery, weight-loss and glycaemic control at the systems

37 level. Bariatric surgery reversed a number of disrupted pathways characteristic of T2D. The

38 differential metabolite set representative of bariatric surgery overlapped with both diabetes (19.3\%

39 commonality) and BMI (18.6\% commonality). However, the percentage overlap between diabetes

40 and $\mathrm{BMI}$ was minimal (4.0\% commonality), consistent with weight-independent mechanisms of T2D

41 resolution. The gut microbiota was more strongly correlated to BMI than T2D, although we identified

42 some pathways such as amino acid metabolism that correlated with changes to the gut microbiota

43 and which influence glycaemic control. Improved understanding of GM-host co-metabolism may lead

44 to novel therapies for weight-loss or diabetes.

\section{Keywords}

48 Gut Microbiome, Gut Microbiota, Bariatric Surgery, Diabetes, Obesity, Roux-en-Y Gastric Bypass, 49 Sleeve Gastrectomy, metabolomic 


\section{Introduction}

52 The global epidemic in obesity and associated disease states carries a significant health and economic 53 burden. The gut microbiota (GM) has been implicated as a contributing factor in a number of these 54 diseases, including obesity and type-2 diabetes (T2D $)^{1,2,3}$. Faecal microbiota transplant experiments 55 in obesity ${ }^{3}$ and $\mathrm{T}^{2} \mathrm{D}^{3,4}$ have shown that this relationship is causal, but these studies have failed to 56 fully unravel the complex mechanisms behind this observation, further complicated by the fact that 57 each individual's GM is unique and subject to redundancy in its metabolic function ${ }^{5}$. Therefore, there 58 is a need to move beyond simply profiling the composition of GM communities in order to understand 59 the true nature of host-microbe relationships.

60 Surgical procedures such as Roux-en-Y gastric bypass (RYGB) and vertical sleeve gastrectomy (VSG) 61 achieve sustainable weight-loss in obesity ${ }^{6}$. Importantly, they are also highly successful in the 62 resolution of obesity-related co-morbidities including $\mathrm{T}^{2} \mathrm{D}^{7}$. These metabolic outcomes are achieved 63 through both weight-dependent and, interestingly, weight-independent mechanisms ${ }^{8}$. Weight64 independent effects occur because bariatric surgery, particularly RYGB, induces a complex system65 wide metabolic effect, including modification of the GM-host metabolic axis ${ }^{9}$. The overwhelming disruption to the GM caused by bariatric surgery is only just being defined, as is its functional importance. To date, few studies have explored longitudinal host-microbe interactions in human cohorts following bariatric surgery, with most studies focussing on either the microbiota or the metabolome ${ }^{10,11}$. Multiple mechanisms for the GMs contribution to achieving weight-loss and metabolic improvement post-surgery have been hypothesised, including: reduced energy harvest of non-digestible food types such as complex carbohydrates; reduced gut permeability leading to decreased systemic inflammation; and alterations in microbe-host co-metabolites such as bile acids (BAs), amino acids (AAs) and short-chain fatty acids (SCFAs) ${ }^{10,12}$.

Bariatric surgery provides a unique opportunity to unravel these complex host-microbe interactions through longitudinal phenotyping before and after intervention to reduce the impact of interindividual variability. Here we have performed multi-platform profiling, to establish changes in the host-microbe interactions in volunteers with obesity +/- T2D and in individuals undergoing bariatric surgery with and without T2D to identify dysregulated pathways in T2D that are functionally restored after bariatric surgery. First, we have compared differences in GM-host co-metabolism in participants with $\mathrm{T} 2 \mathrm{D}$ compared to non-diabetic individuals at baseline to ascertain which metabolites were 
81 associated with glycaemic control. Next, we profiled subgroups of patients undergoing RYGB or VSG

82 to evaluate changes in GM-host co-metabolism following these contrasting interventions and

83 assessed their impact on glycaemic control, taking into account intervention-dependent changes in

84 eating behaviour.

\section{Results}

86 Serum samples were collected from 158 participants with obesity. Sixty-six participants had T2D and

8726 had Impaired Glucose Tolerance (IGT). Complete sample sets of serum, 24-hour urine and stool

88 were collected from 81 of these individuals (42 T2D, 11 IGT).

89 Forty-nine patients underwent bariatric surgery (VSG=26, RYGB=23; Figure 2A) and gave serum 90 samples pre and 3-months post-surgery (19 T2D, 6 IGT). Twenty-seven of these participants (VSG=14, 91 RYGB =13) gave complete sample sets of serum, 24-hour urine and stool pre and 3-months post92 surgery. More diabetic patients underwent RYGB than VSG (11/23 vs 8/26). Otherwise, baseline 93 demographics were not significantly different between procedures. Full demographics are detailed 94 in the Supplemental Information R1.

95 Microbial and metabolic profiling indicated systematic differences relating to obesity, T2D and 96 bariatric surgery (both RYGB and VSG), with metabolic signatures identified across the three biofluids 97 (urine, serum, faecal water). Each condition had a specific set of metabolic correlates, with some 98 overlap between groups. We identified 207 metabolites associated with bariatric surgery, 54 (26\%) 99 of these metabolites were characteristic of improved glycaemic control, $41(20 \%)$ associated with $100 \mathrm{BMI}$ reduction and 28 (14\%) were associated with dietary changes (Figure 1). Consistent with the 101 observation that the mechanism for T2D resolution following bariatric surgery is partially 102 independent of weight-loss, of the 175 metabolites associated with either T2D or BMI only 7 103 overlapped ( $4 \%$ commonality). 


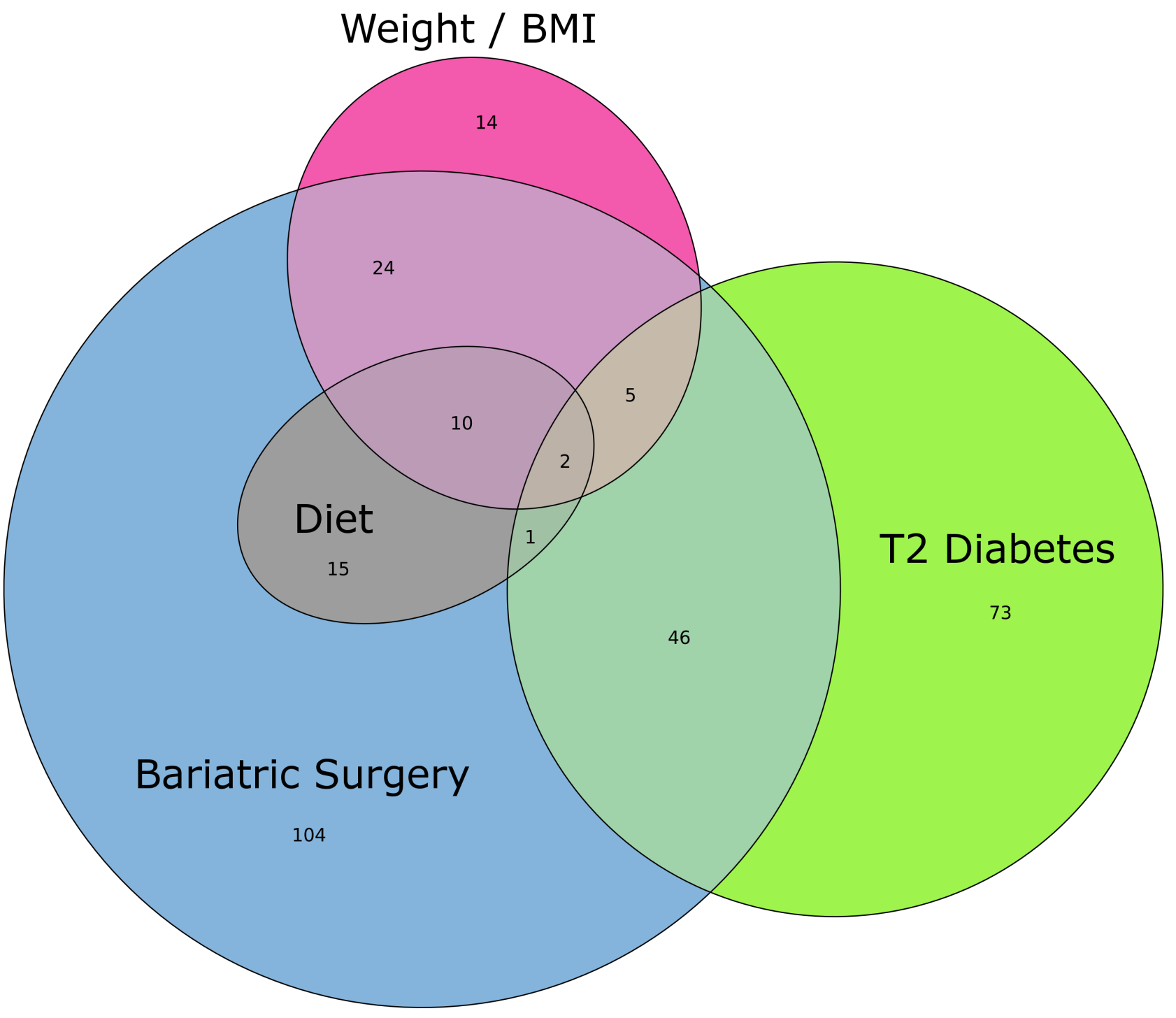

Figure 1. Euler diagram of identified metabolites associated with Bariatric Surgery, Weight / BMI, T2D and Diet.

108 Euler diagram of identified metabolites from serum, urine and faecal biofluids with associations 109 (pFDR<0.05) to 1) post-versus pre-bariatric surgery, 2) lower weight / BMI, 3) lower HbA1c / non-T2D 110 Vs T2D, 4) lower dietary substrate / higher Alternative Healthy Eating Index (AHEI-2010) score. 111 Metabolites with concordant changes are grouped together. Metabolites from each grouping and 112 their associations are detailed in Supplemental Information R2. 
114 Gut microbial differences between T2D and non-diabetic individuals

115 Shotgun metagenomic profiling did not identify a difference in microbial gene richness or $\beta$-diversity 116 of the GM derived from the distal colon between T2D and non-diabetic participants at baseline.

117 However, compositional analysis of the GM demonstrated lower relative abundance of the genera 118 Escherichia (Proteobacteria), Peptostreptococcaceae (Firmicutes) and Barnesiella (Bacteroidetes) in 119 T2D relative to non-diabetic controls. Individual species from the genera Ruminococcus, 120 Parabacteroides and Bacteroides had higher relative abundance (Figure 3D). Functional analysis of 121 the GM found lower levels of KEGG pathways relating to cofactor and vitamin metabolism, including 122 nicotinate and nicotinamide metabolism and one-carbon metabolism by folate in T2D relative to non123 diabetic controls. Methane metabolism, streptomycin and neomycin biosynthesis, polycyclic 124 aromatic hydrocarbon degradation and $D$-alanine metabolism pathways were also less prevalent in 125 T2D, while bisphenol degradation pathways were higher.

\section{Metabolic differences between T2D and non-diabetic individuals}

128 Individuals with T2D were metabolically distinct from non-diabetic participants as determined from 129 both targeted MS assays and global ${ }^{1} \mathrm{H}-\mathrm{NMR}$ profiles. In participants with T2D, the serum secondary 130 to primary (2:1) BA ratio was higher compared to non-diabetics at baseline. Conjugation of the 131 primary BA cholic acid (CA), the overall glycine:taurine conjugation ratio of primary BAs and 132 lithocholic acid were also higher in T2D. Conversely, CA and the CA:CDCA (chenodeoxycholic acid) 133 ratio were lower. In addition, conjugation of secondary BAs including tauro-ursodeoxycholic acid 134 (TUDCA), taurohyocholic acid (THCA), glycohyocholic acid (GHCA) and the conjugated UDCA:UDCA 135 ratio was lower. In faeces, $5 \alpha$-cholanic acid-3 $\alpha$-ol-6-one was lower in individuals with T2D relative to 136 non-diabetic controls. Targeted GC-MS analysis found higher 2-hydroxybutyrate and lactate in T2D 137 participants' urine and serum relative to controls. Serum 2-methylbutyrate and isovalerate were also 138 higher in T2D participants, while urinary butyrate was lower. Further quantitative analyses of serum 139 metabolites found higher branched-chain AAs (BCAAs) leucine, isoleucine and valine, aromatic AAs 140 (AAAs) phenylalanine and tyrosine, as well as alanine, methionine, glutamate, lysine and proline in 141 volunteers with T2D relative to non-diabetic controls. The biogenic amines 2-aminoadipate, 142 methionine sulfoxide and sarcosine and short-chain acylcarnitines (C2-5, 9) were also present in 143 higher concentrations. Conversely, longer-chain acylcarnitines $(C 14,16,18)$ and a number of lyso- 
144 phosphatidylcholines, acyl-alkyl-phosphatidylcholines, longer-chain diacyl-phosphatidylcholines and 145 sphingomyelins were present in lower concentrations in T2D.

146 Orthogonal Partial Least Squares - Discriminant Analysis (OPLS-DA) models of global ${ }^{1} \mathrm{H}-\mathrm{NMR}$ spectra 147 comparing those with and without T2D were generated. Serum and faeces produced robust models $148\left(R^{2} Y=0.61, Q^{2} Y=0.44\right.$ and $R^{2} Y=0.59, Q^{2} Y=0.35$ respectively) while urine produced the least robust 149 model $\left(R^{2} Y=0 \cdot 80, Q^{2} Y=0 \cdot 12\right)$. In serum, participants with $T 2 D$ were characterised by higher 150 concentrations of VLDL/LDL lipoproteins, BCAAs, lactate, alanine, proline, pyruvate, tyrosine and $\alpha$ 151 glucose relative to non-diabetic controls, whereas HDL, glutamine, glycerophosphocholine, 152 phosphocholine/choline and histidine were lower. In faeces, individuals with T2D had higher levels 153 of glycine and the anti-hyperglycaemia drug dimethylbiguanide (Metformin). Lactate, uracil, BCAAs, 154 and tyrosine were lower relative to non-diabetic controls. As expected, in urine, $\alpha$ - and $\beta$-glucose 155 were higher in T2D participants, while isobutyrate, glycine, creatine, creatinine, $\mathrm{O}$-acetylcarnitine, $\mathrm{N}$ 156 methyl-2-pyridone-5-carboxamide, methylnicotinamide and formate were lower relative to non157 diabetic controls.

Integrative analysis of metabolic and gut microbiota profiles in T2D and non-diabetic individuals

161 Multi-omic signatures of participants with T2D versus non-diabetic individuals were modelled using 162 Data Integration Analysis for Biomarker discovery using Latent cOmponents (DIABLO), with a crossvalidated balanced error rate (BER) of 0.18 , indicating good class separation at the systems level (see

164 Supplemental Information R4). Volunteers with T2D were characterised by higher levels of lactate, glucose and alanine in serum; lactate, glucose and 2-hydroxybutyrate in urine and dimethylbiguanide (Metformin) in faeces compared to non-diabetic controls. Whereas lower serum phosphatidylcholines and HDL lipoprotein; lower urinary glycine, trimethylamine and isobutyrate and faecal valine and uracil levels were characteristic of T2D participants relative to non-diabetics. Lower levels of GM from the Peptostreptococcaceae (Firmicutes) and Barnesiella (Bacteroidetes) genera

171 lipid and $\mathrm{N}$-glycan biosynthesis; nicotinate, nicotinamide, methane, alanine and one-carbon 172 metabolism; and biosynthesis of secondary metabolites such as streptomycin and neomycin. 


\section{Clinical findings after bariatric surgery}

175 Significant weight-loss was achieved after both procedures (Figure 2B) although the percent weight176 loss was greater after RYGB $(p=0.023)$ (Figure 2B). At 3-months post-surgery T2D patients who 177 underwent VSG and RYGB had mean ( \pm standard deviation) glycated haemoglobin (HbA1c) reductions 178 of $17 \cdot 8 \mathrm{mmol} / \mathrm{mol}( \pm 11 \cdot 2)$ and $19.4 \mathrm{mmol} / \mathrm{mol}( \pm 12 \cdot 5)$ respectively. Relative to baseline, VSG and RYGB 179 patients had reductions in $\mathrm{HbA} 1 \mathrm{c}$ of $27 \cdot 6 \%( \pm 12 \cdot 1)$ and $28 \cdot 6 \%( \pm 14 \cdot 2)$ respectively. Three months 180 after VSG, 4/8 T2D participants had complete diabetes resolution ( $\mathrm{HbA} 1 \mathrm{c}<42 \mathrm{mmol} / \mathrm{mol}$ ), $2 / 8 \mathrm{had}$ 181 partial resolution $(\mathrm{HbA} 1 \mathrm{c}<48 \mathrm{mmol} / \mathrm{mol}$ ) and $2 / 8$ had ongoing T2D ( $\mathrm{HbA} 1 \mathrm{c}>48 \mathrm{mmol} / \mathrm{mol})$. Of 4 182 participants with IGT that underwent VSG, two had resolution. Three months after RYGB, 8/11 T2D 183 participants had complete diabetes resolution and 3/11 had ongoing T2D. Two participants with IGT 184 underwent RYGB, both had complete resolution.

185 Three months following surgery there were large changes in dietary intake compared to baseline. As 186 measured by three self-reported 24-hour dietary recall questionnaires collected at each time point, 187 there were significant reported reductions in calorie, carbohydrate, fat, fibre and sodium intake after 188 both operations. In addition, protein intake was significantly reduced after VSG, while saturated fat 189 and sugar intake were significantly reduced after RYGB (Figure 2C). Dietary healthiness, measured 190 using the Alternative Healthy Eating Index (AHEI-2010) ${ }^{13}$, was increased 3-months after RYGB and 191 VSG surgery (median 42 vs $54, p=0 \cdot 002$, Figure 2D). 
A
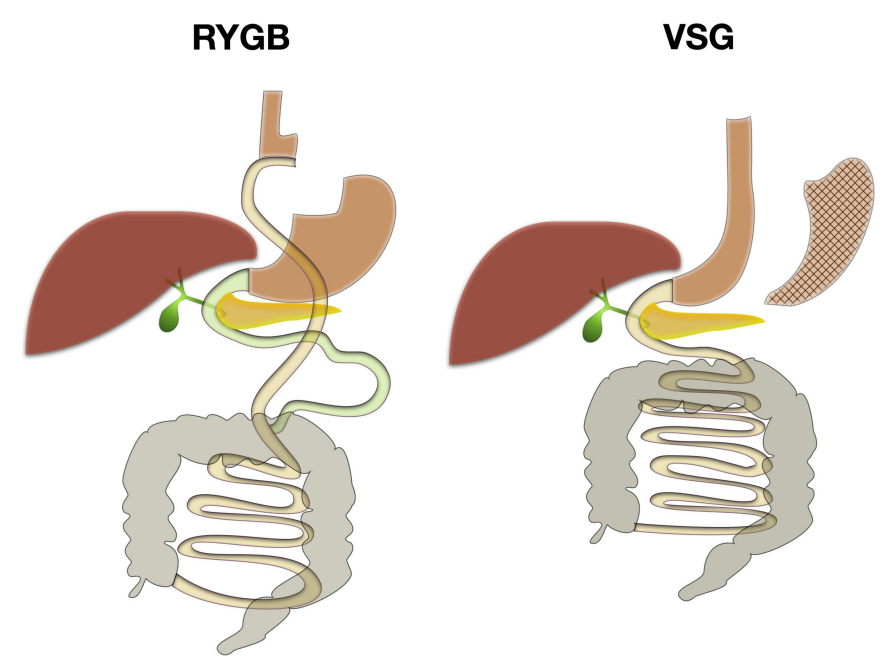

C

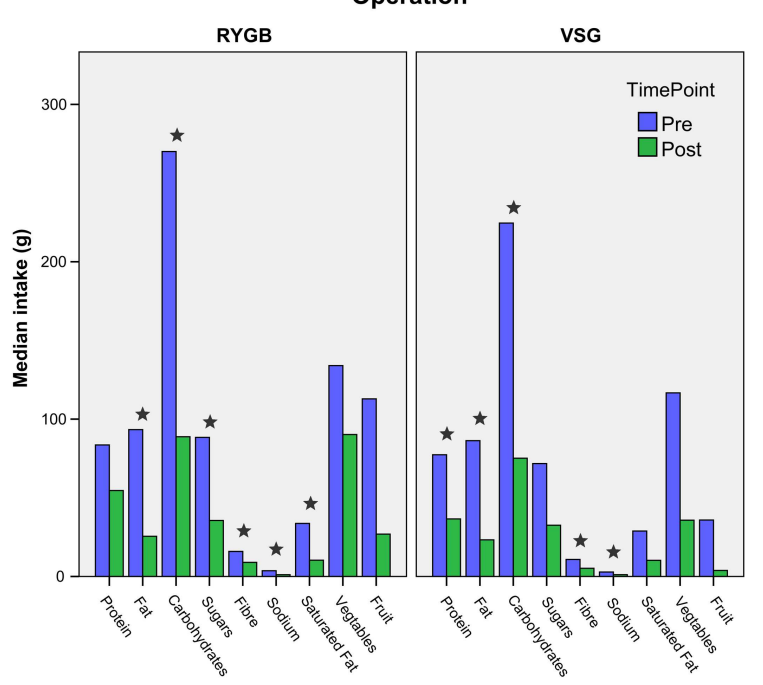

B

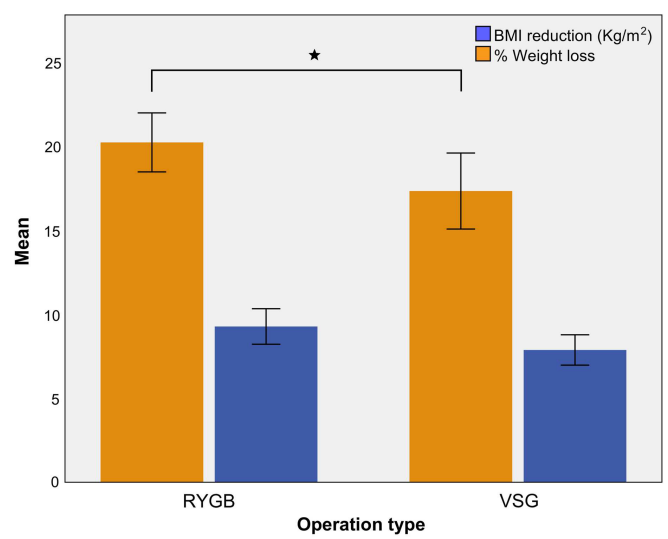

D

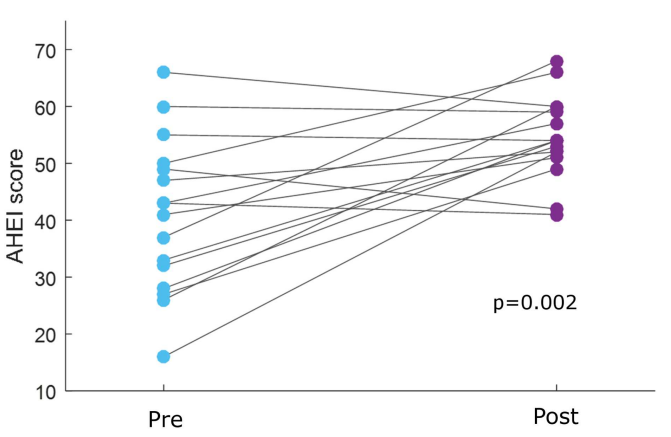

194 Figure 2. Clinical findings after bariatric surgery.

195 (A) Schematic of Roux-en-Y Gastric Bypass (RYGB) and Vertical Sleeve Gastrectomy (VSG) procedures, 196 showing the respective anatomical changes. (B) Weight-loss 3-months post RYGB ( $n=23)$ and VSG 197 (n=26) procedures. Patients who underwent VSG had a mean (SD) weight-loss, percent weight-loss 198 and $B M I$ reduction of $21.7 \mathrm{~kg}(6 \cdot 8), 17 \cdot 3 \%(5 \cdot 6)$ and $7.9 \mathrm{~kg} / \mathrm{m} 2(2 \cdot 2)$ respectively. Patients who 199 underwent RYGB had a mean (SD) weight-loss, percent weight-loss and BMI reduction of $24 \cdot 6 \mathrm{~kg}(7 \cdot 0)$, $20020 \cdot 2 \%(4 \cdot 1)$ and $9 \cdot 3 \mathrm{~kg} / \mathrm{m}^{2}(2 \cdot 4)$ respectively. 95\% confidence intervals are shown. Percent weight-loss 201 was significantly greater in the RYGB group ( $p=0.02)$. (C) Dietary changes 3-months post RYGB and 202 VSG procedures. (D) Dietary healthiness measured by the Alternative Healthy Eating Index (AHEI) pre 203 and 3-months post bariatric procedures. ${ }^{*}=p<0.05$. 
206 Multivariate analysis demonstrated a change in $\beta$-diversity (Bray-Curtis dissimilarity) after RYGB 207 (PERMANOVA $p=0 \cdot 002$ ), but not VSG (Supplemental Information R3). There was no difference in 208 microbial gene richness after either bariatric procedure. Compositional analysis revealed a major 209 disruption to the GM after RYGB, but more subtle changes after VSG. Three months after RYGB, 210 participants had: i) Increased relative abundance of Veillonella, Gemella, Granulicatella, 211 Enterococcus, Streptococcus and Clostridium (Firmicutes), Fusobacterium (Fusobacteria), Klebsiella 212 and Escherichia (Proteobacteria), Actinomyces and Anaerotruncus (Actinobacteria) and Prevotella 213 (Bacteroidetes); ii) decreased relative abundance of Holdemania, Eubacterium, Faecalibacterium, 214 Subdoligranulum, Ruminococcus, Dorea and Anaerostipes (Firmicutes), Burkholderiales 215 (Proteobacteria), Bifidobacterium and Collinsella (Actinobacteria). Changes at each taxonomic rank 216 from phylum to species are shown in Figure 3A. Functional analysis found an increase in bacterial 217 KEGG pathways pertaining to: AA metabolism, lipid metabolism including fatty acid degradation, $\alpha$ 218 linolenic acid metabolism; and xenobiotic biodegradation including benzoate, aminobenzoate and 219 ethylbenzene degradation. Bile salt hydrolase (choloylglycine hydrolase) pathways were reduced $220(p=0 \cdot 03)$ (Figure 3B).

221 Three months after VSG, participants had increased relative abundance of a select number of species 222 within the genera Streptococcus, Eubacterium and Anaerotruncus (Firmicutes) and Escherichia 223 (Proteobacteria) and decreased species within Faecalibacterium, Dorea, Anaerostipes, Roseburia and 224 Coprococcus (Firmicutes) (Figure 3C). Limited changes to KEGG pathways were found after VSG. 
A) Differentially Abundant Taxa Post Roux-en-Y Gastric Bypass

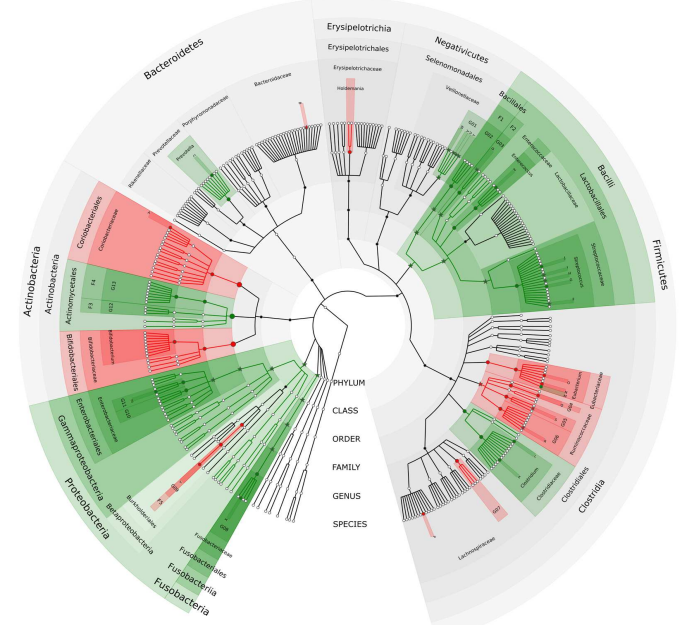

C) Differentially Abundant Taxa Post Sleeve Gastrectomy

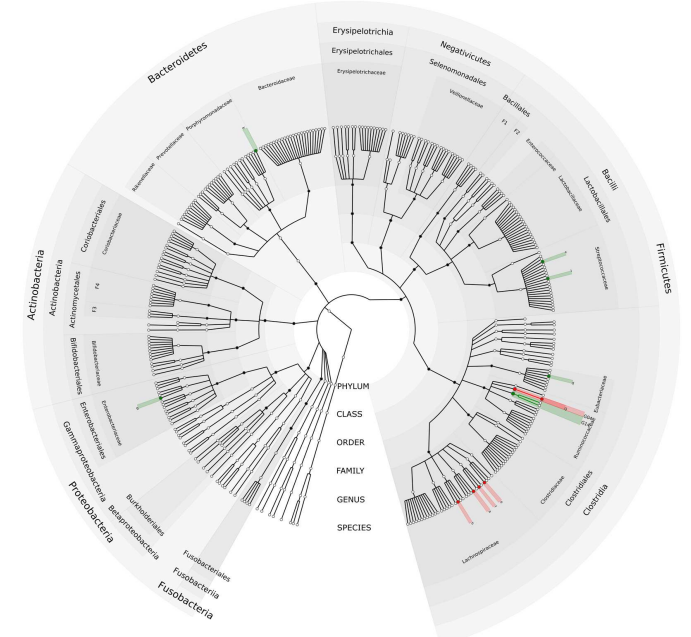

B) Differentially Abundant KEGG Pathways Post RYGB

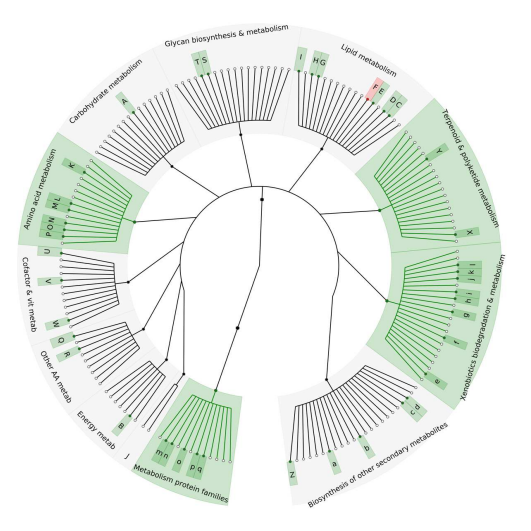

D) Differentially Abundant Taxa T2D Vs Non-Diabetic

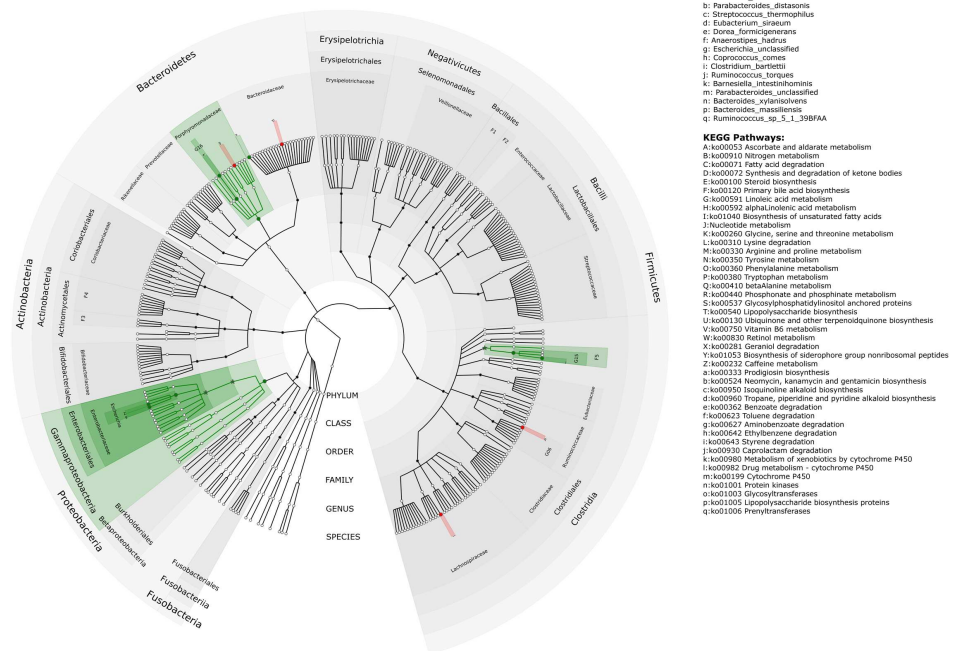

227 Figure 3. Gut microbiota changes after bariatric surgery and in T2D vs non-diabetic controls.

(A) Phylogenetic tree of significant differentially abundant taxa from phyla to species 3-months post RYGB. (B) Phylogenetic tree of significant differentially abundant level 2 and level 3 KEGG pathways 3-months post RYGB. (C) Phylogenetic tree of significant differentially abundant taxa from phyla to species 3-months post VSG. (D) Phylogenetic tree of significant differentially abundant taxa from phyla to species between T2D and non-diabetic participants. Taxa / KEGG pathways significantly increased post-surgery (A-C) or lower in T2D vs non-diabetic (D) are shown in green, taxa / KEGG pathways significantly decreased post-surgery $(A-C)$ or higher in T2D vs non-diabetic $(D)$ are shown in red. Changes that remain significant after Benjamini-Hochberg multiple testing corrections are denoted with an asterisk (*). Plots A-D are shown individually in Supplemental Information R3. 
239 After RYGB and VSG, both groups had increased secondary (UDCA) and conjugated secondary BAs 240 (GUDCA+TUDCA) in serum. Increases in glycine conjugation relative to taurine in both primary and 241 secondary serum BAs occurred after RYGB. Whereas decreased primary and conjugated primary BAs 242 were noted after VSG. Additionally, GHCA was increased after RYGB, while murocholic acid and 243 isolithocholic acid were increased after VSG. Faecal levels of primary, secondary, conjugated primary 244 and secondary and overall faecal BAs were decreased after both procedures. Similar to serum there 245 was an increase in the conversion ratio of primary to secondary BAs in faeces. Absolute $5 \alpha$-cholanic 246 acid-3 $\alpha$-ol-6-one and allolithocholic acid were increased after RYGB and VSG respectively. 5 3 247 cholanic acid-3 $\alpha$-ol-12-one and 3,6/3,12-diketocholanic acid were reduced after RYGB. After RYGB, 248 but not VSG, excretion of 2-methylbutyrate and isovalerate in urine and faeces increased. In serum, 249 lactate was decreased after RYGB and showed a non-significant trend towards decreasing after VSG, 250 consistent with the lower concentrations of serum lactate in non-diabetics, compared to diabetics. 251 In faeces, acetate was reduced after both procedures, while butyrate and valerate reduced after VSG. 252 Both procedures resulted in a decrease in the majority of serum AAs, including BCAAs and AAAs. 253 However, glycine and serine were increased after both procedures, with glutamine also increased 254 after RYGB. Serum kynurenine and sarcosine were decreased and symmetric dimethylarginine was 255 increased after both procedures. 2-Aminoadipate was lower after VSG. Short-chain acylcarnitines 256 (C0, C3-5) and a large number of phosphatidylcholines and lyso-phosphatidylcholines species were 257 reduced after both RYGB and VSG. Whereas, C2 and longer-chain acylcarnitines $(C 7,8,10,14,16$, 258 18), sphingomyelins and predominantly longer-chain acyl-alkyl-phosphatidylcholines were increased, 259 with similar findings after both interventions.

260 Repeated Measures, Monte-Carlo Cross-Validation, Partial Least Squares Discriminant Analysis (RM261 MCCV-PLSDA) models of serum and urine global ${ }^{1} \mathrm{H}-\mathrm{NMR}$ spectra found excellent separation between 262 participants pre- and post-surgery, with robust models after both procedures (Figure 4). In serum, a 263 number of significant changes were consistent after both RYGB and VSG, including altered AA 264 metabolism, matching results in the targeted AA analysis. Ketone bodies acetone, acetoacetate and 265 3-hydroxybutyrate were increased and evidence of changes to the tricarboxylic acid cycle was seen, 266 with citrate increasing and pyruvate decreasing after surgery. VLDL/LDL, lipid glycerol and choline 267 decreased 3-months after RYGB and VSG. In addition, lactate decreased after RYGB, consistent with 268 the quantitative GC-MS analysis. In urine, a number of significant metabolic changes were also 
269 consistent after both RYGB and VSG procedures, including increased bacterially derived metabolites 270 such as phenylacetylglutamine (PAG), N-methyl-4-pyridone-3-carboxamide, 4-cresylsulfate, 271 hippurate, trimethylamine- $N$-oxide (TMAO) and 2-aminobutyrate. Decreased urinary excretion of 272 lactate, 3-hydroxyisovalerate and AAs occurred and a reduction in analgaesic use was observed. RM273 MCCV-PLSDA models of faecal samples analysed by ${ }^{1} \mathrm{H}-\mathrm{NMR}$ pre- and post-surgery produced a robust 274 model for VSG but not RYGB (Supplemental Information R2). Tyramine and $\beta$-alanine were increased 275 after VSG. In keeping with the quantitative SCFA analysis, acetate, butyrate, valerate were decreased, 276 as were isovalerate, lactate, methanol, formate, trimethylamine and phenylacetate.

277 Full details of metabolic changes can be found in Supplemental Information R2. 


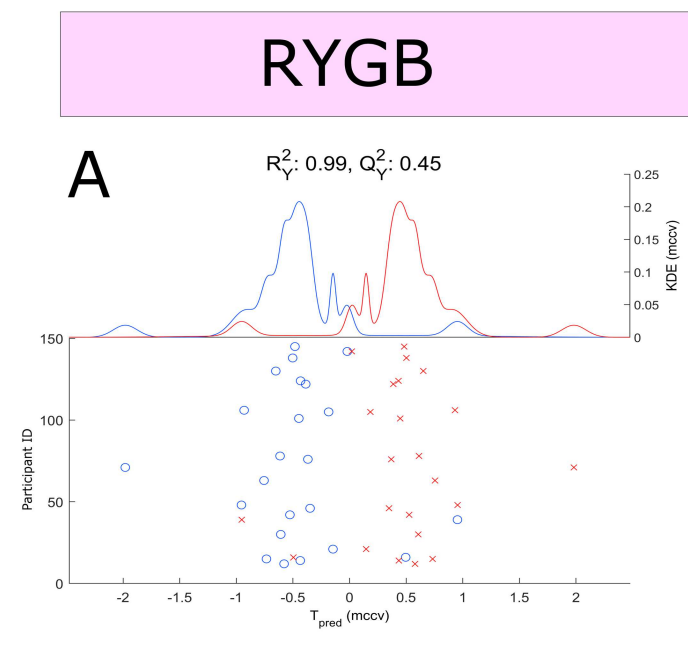

\section{VSG}
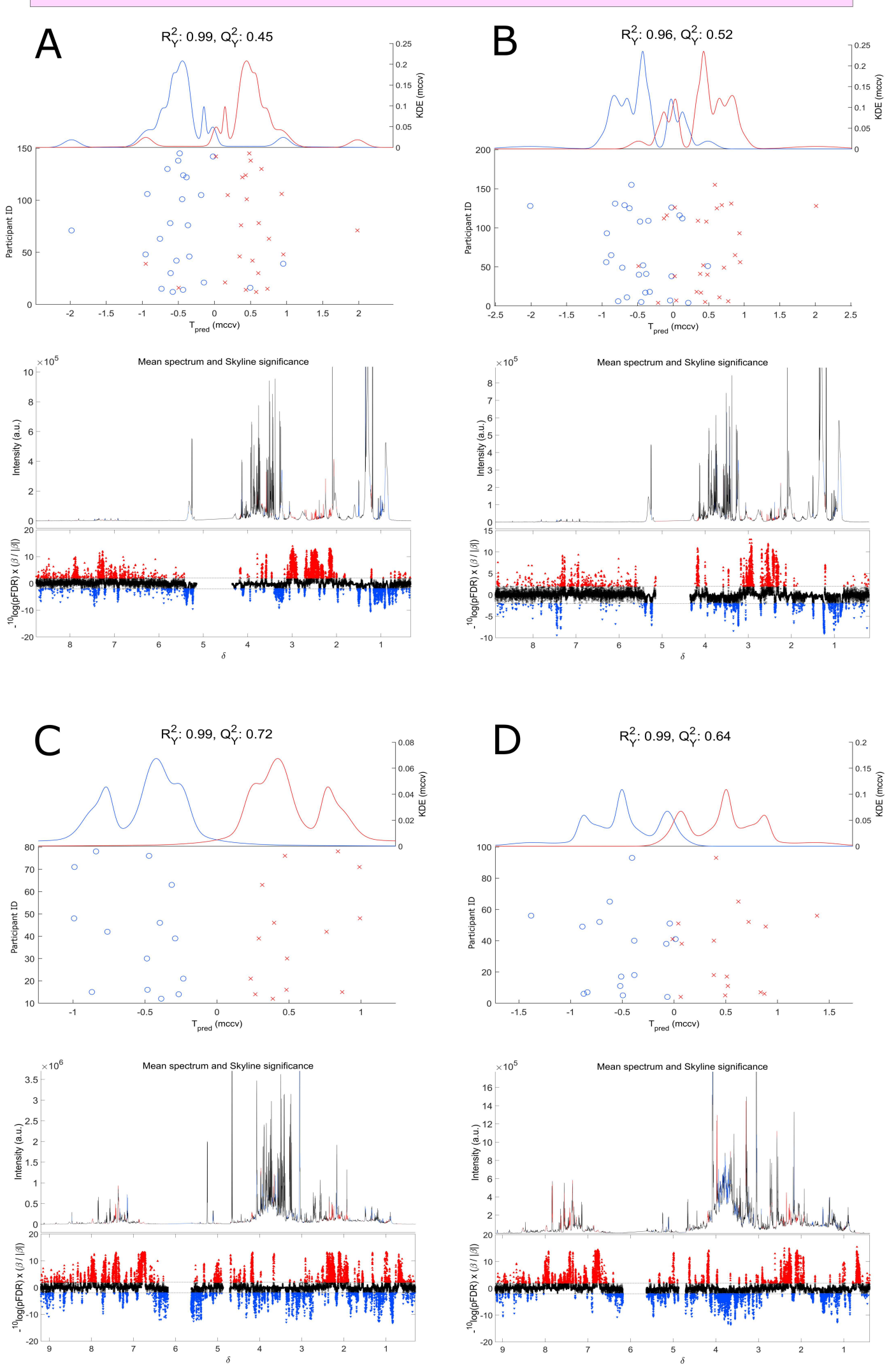

O
$\frac{1}{3}$
$\frac{3}{3}$
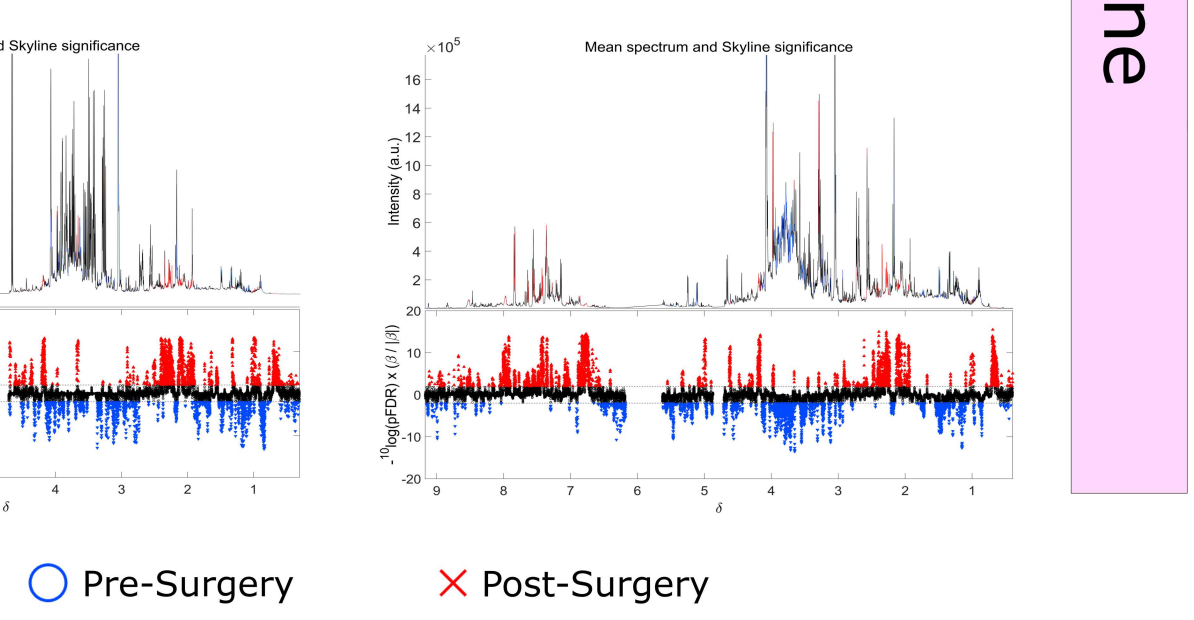

X Post-Surgery 
Figure 4. Metabolite changes after RYGB and VSG procedures, assessed by ${ }^{1} H-N M R$.

RM-MCCV-PLSDA models were generated using ${ }^{1} H-N M R$ spectra derived from serum and urine biofluids pre and 3-months post RYGB and VSG procedures. Model scores: (A) Serum RYGB ( $n=23, R^{2} Y$ 0.99, $\left.Q^{2} Y 0.45\right)$, (B) Serum VSG ( $\left(n=26, R^{2} Y 0.96, Q^{2} Y 0.52\right)$, (C) Urine RYGB ( $\left.n=14, R^{2} Y 0.99, Q^{2} Y 0.72\right)$, (D) Urine VSG ( $\left.n=16, R^{2} Y 0.99, Q^{2} Y 0.64\right)$ ), (Supplemental Information R2) Faeces $R Y G B\left(n=10, R^{2} Y 0.94\right.$, $\left.Q^{2} Y 0.06\right)$, (Supplemental Information R2) Faeces VSG ( $\left.n=14, R^{2} Y 0.98, Q^{2} Y 0.66\right)$. Upper panels: $R M$ $M C C V$-PLSDA scores plots comparing participant samples pre and 3-months post bariatric surgery. Models are comprised of 1 predictive and 1 orthogonal component. Lower panels: Mean ${ }^{1} \mathrm{H}-\mathrm{NMR}$ spectrum and Manhattan plot. Manhattan plot showing - $\log _{10}($ pFDR $)$ x sign of the variable regression coefficient for each variable within the RM-MCCV-PLSDA model. Dotted lines illustrate the pFDR significance cut off level (0.01) on the $\log _{10}$ scale. Spectra considered significant are highlighted in the Manhattan plot and mean spectrum. Red metabolites $\left({ }^{1} H\right.$-NMR signals) are significantly increased post-surgery, blue metabolites ( ${ }^{1} \mathrm{H}$-NMR signals) are significantly decreased post-surgery.

\section{Integrative analysis of metabolic and gut microbiota profiles after bariatric surgery}

Multi-omic signatures of the response to RYGB and VSG were modelled using multilevel DIABLO, with cross-validated balanced error rates (BER) of 0.021 and 0.085 respectively, indicating significant differences at the systems level in response to RYGB and VSG and excellent classification between pre- and post-surgery states (Figure 5A-F). Each procedure had a distinct signature. Notably, RYGB was characterised by an increase in tyrosine and phenylalanine metabolism and benzoate and fatty acid degradation pathways in gut bacteria. This difference corresponded with increases in urinary metabolites of bacterial origin including PAG, hippurate and TMAO. In addition, there was increased serum glycine and glycine conjugation of $\mathrm{CA}$ and decreased tryptophan and valerate. A number of $\mathrm{BA}$ changes were seen in faeces including increased glyco-ursocholanic acid and $5 \beta$-cholanic acid $3 \alpha$-ol6-one. VSG was characterised by a decrease in a number of GM KEGG pathways. These correlated with decreased faecal SCFA levels, specifically acetate, butyrate and valerate. However, similar to RYGB a number of bacterially derived compounds were increased in the urine including indoxylsulfate, 4-cresylsulfate, hippurate and PAG. Serum changes consisted predominantly of decreased phosphatidycholines.

A multi-omic signature differentiating between the two bariatric procedures was characterised by a greater increase in urinary compounds of microbial origin including PAG, indoxylsulfate, TMAO and 4-hydroxybutyrate after RYGB relative to VSG. The reduction in faecal acetate, butyrate, valerate was greater after VSG, while an increase in 2-methylbutyrate and isovalerate was specific to RYGB. Glycine conjugation of serum BAs was greater after RYGB. A number of bacterial species increased 
314 further after RYGB, including Escherichia coli and unclassified Granulicatella and Gemella species, as 315 did GM KEGG pathways including tryptophan metabolism, benzoate and toluene degradation and 316 biosynthesis of unsaturated fatty acids (Supplemental Information R4).

317

A
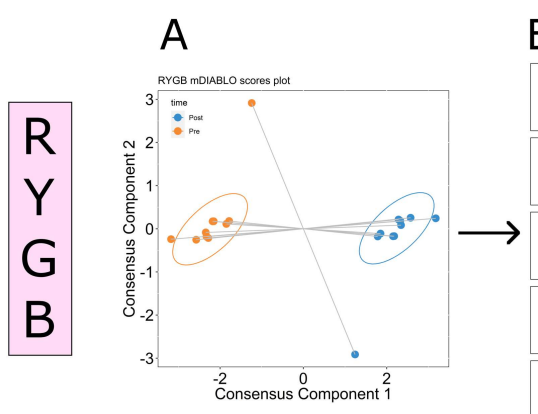

B
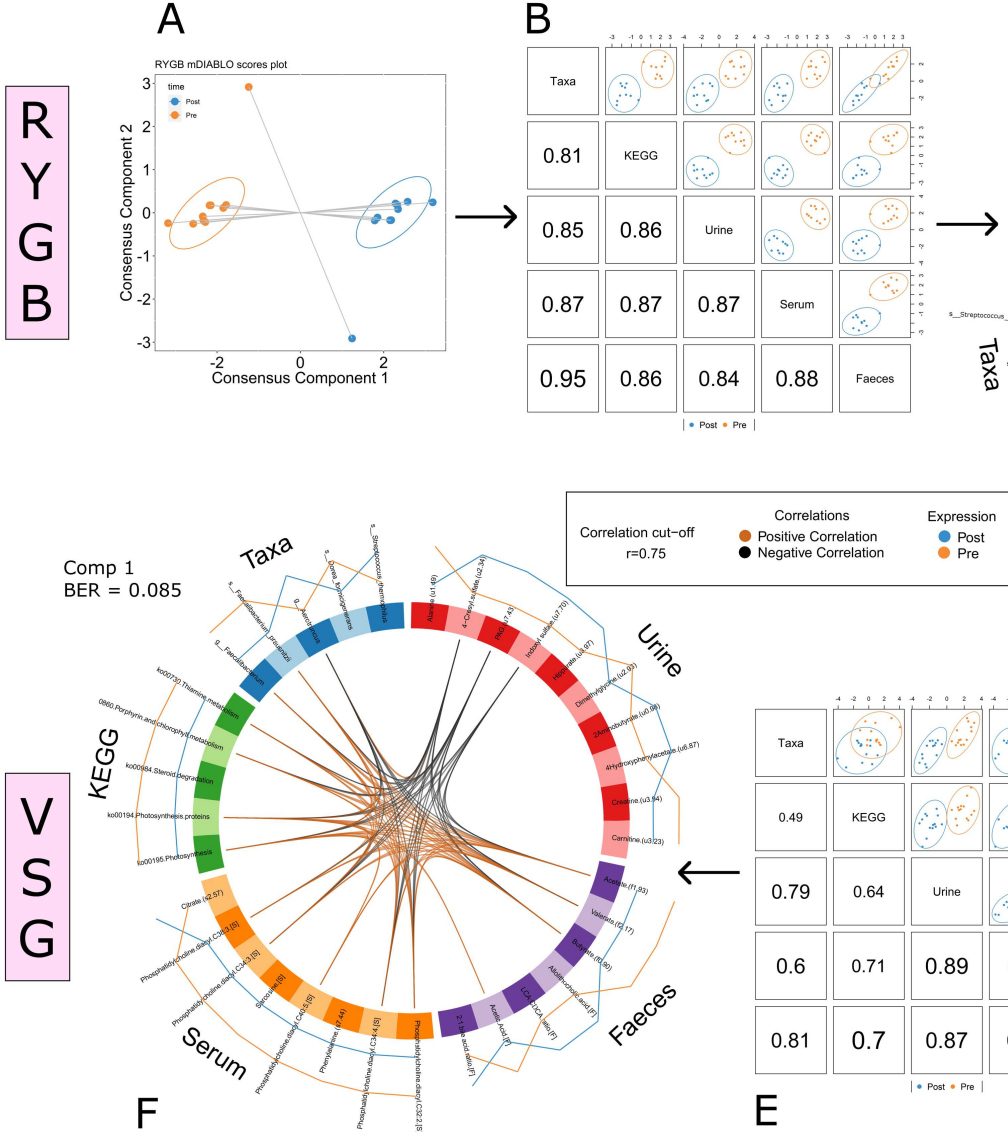

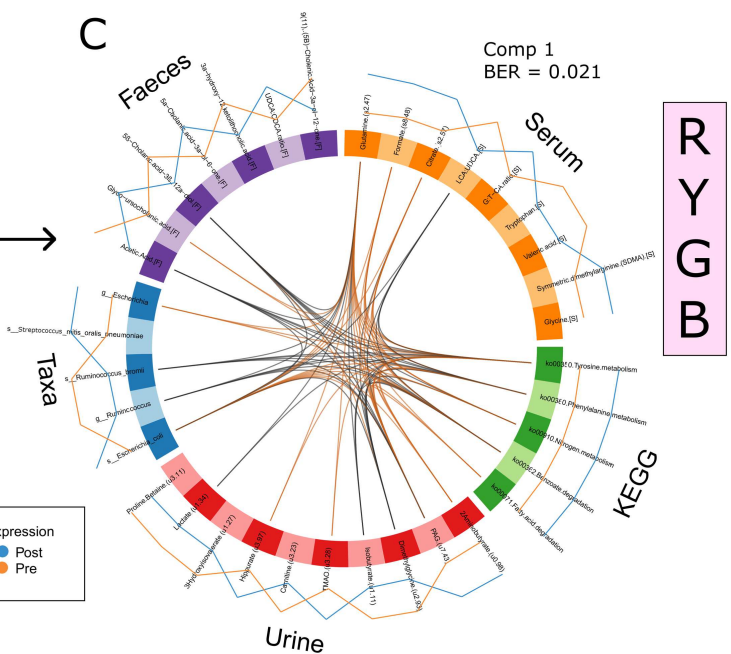

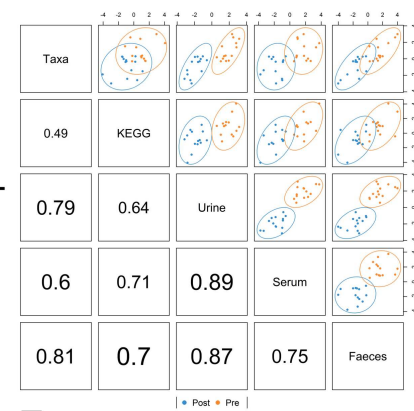

$\mathrm{E}$

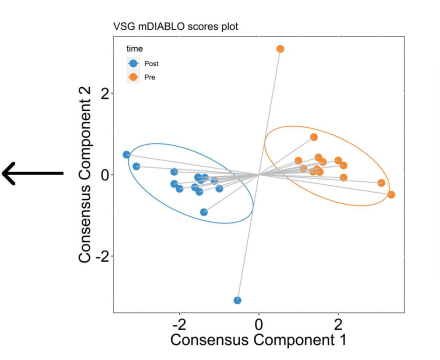

D

319 Figure 5. Integration of metabolite and gut microbiota datasets.

Multi-omic datasets pre and 3-months post RYGB (A-C) and VSG (D-F) surgery were integrated using multilevel Data Integration Analysis for Biomarker discovery using Latent cOmponents (mDIABLO). $(A, D)$ Scores plots with samples projected in latent space for RYGB $(n=10)$ and VSG $(n=14)$ models respectively. Classes (pre-/post-surgery) are discriminated along component 1. (B, E) Scores plots derived from component 1 of individual datasets, showing correlations between variables from each dataset. (C, F) Variables from component 1 (coloured by dataset) discriminating between pre/post timepoints are displayed. Differences in expression of variables pre-/post-surgery are shown by the colour-coded outer lines. Correlations $(r>0.75)$ between variables are shown by the colour-coded inner lines. Cross-validated balanced error rates (BER) for each model are shown. Ellipses correspond to 95\% confidence intervals. 
332 Body Mass Index (BMI) is closely associated with glycaemic control (HbA1c) and both decreased 333 following bariatric surgery. However, interestingly, BMI and $\mathrm{HbA1c}$ correlation networks had 334 contrasting compositions (Figure 6). BMI was strongly correlated to a number of bacterially derived 335 factors. Species including Escherichia coli, Streptococcus anginosus, Streptococcus parasanguinis, 336 Clostridium hathewayi and multiple Veillonella species were correlated with a lean phenotype, while 337 Eubacterium rectale was correlated with increased adiposity. Subsequently a number of GM KEGG 338 pathways such as tryptophan metabolism, linoleic acid metabolism, cytochrome P450, steroid 339 biosynthesis and xenobiotic degradation were also correlated with lower BMI. Bacterially derived 340 urinary metabolites PAG, 4-cresylsulfate, indoxylsulfate and 4-hydroxyphenylacetate were negatively 341 correlated with BMI. While SCFAs acetate and valerate in faeces and isobutyrate in urine correlated 342 positively, as did a number of BA species.

343 Conversely, HbA1c correlated positively with a range of serum AAs including BCAAs, acylcarnitines $344(\mathrm{C} 3, \mathrm{C} 5, \mathrm{C} 5-\mathrm{OH})$, lactate, kynurenine and 2-aminoadipate. In validation of the model, $\mathrm{HbA} 1 \mathrm{c}$ increased 345 with higher serum and urinary glucose, faecal metformin levels and age, as expected. Whereas serum $346 \mathrm{HDL}$, sphingomyelins, glycerophophocholine and GHCA, urinary 2-aminobutyrate and glycine and 347 faecal $5 \alpha$-cholanic acid $3 \alpha$-ol-6-one levels were negatively associated with HbA1c.

A

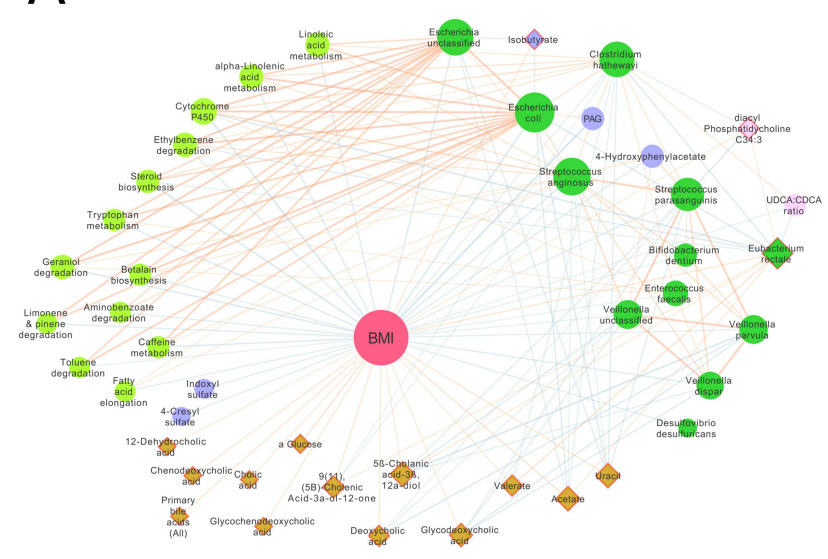

B

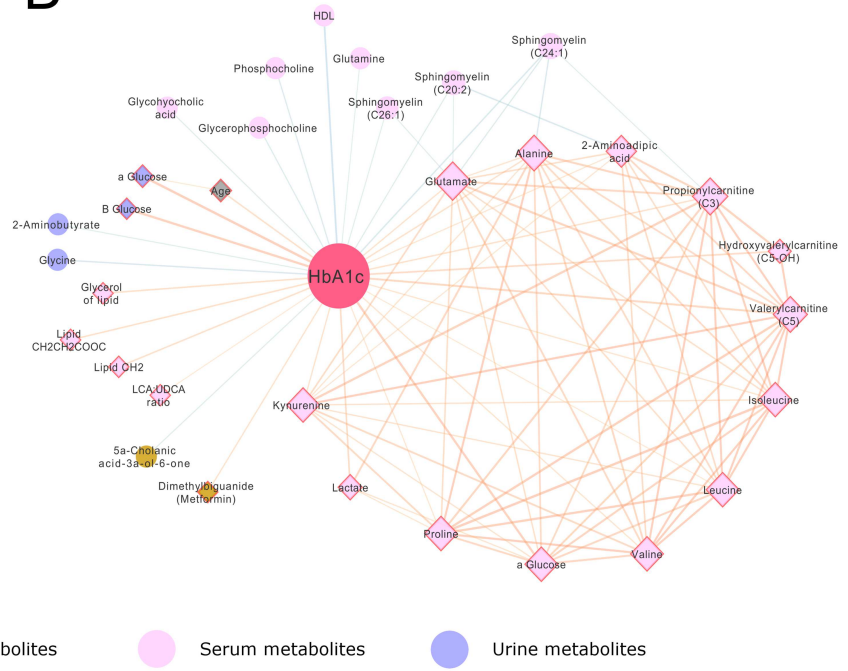

Figure 6. Metabolite and gut microbiota correlations with BMI and HbA1c. 
First-order Spearman's correlations to $B M I(A)$ and $H b A 1 C(B)$ derived from metabolite and microbiota datasets. Correlations with a corrected pFDR $<0.01$ are shown. Variables with a negative correlation to $B M I(A)$ and $H b A 1 C(B)$ are displayed within a circle, variables with a positive correlation have a diamond shape and red outline. Node size is proportional to the number of significant correlations to that variable. Positive correlations between variables are shown with orange lines, negative correlations have blue lines. Line thickness is proportional to the correlation strength ( $r$ ).

\section{Influence of diet on gut microbial and metabolic profiles}

Metabolic changes associated with diet overlapped principally with the effects of bariatric surgery and BMI, but not T2D (Figure 1). Carbohydrate and calorie intake correlated positively with a number of serum glycerophospholipids and sphingomyelins as well as phenylalanine, hydroxypropionylcarnitine $(\mathrm{C} 3-\mathrm{OH})$, propenoylcarnitine $(\mathrm{C} 3: 1)$ and faecal acetate levels (see Supplemental Information R4). While urinary PAG, 4-cresylsulfate and serum acetate were negatively correlated with carbohydrates and calorie intake. PAG was also negatively correlated with fat intake, including trans and polyunsaturated fats. Serum glutamate was higher with higher levels of trans fat and tauro-ursodeoxycholic acid (TUDCA) in serum was negatively correlated with salt (sodium) intake. Fibre intake was positively correlated with serum proline and C24:1-OH sphingomyelin levels. While serum leucine levels were lower as diet healthiness (AHEI-2010 score) increased. Limited changes to the GM relating to diet were identified, but percentage carbohydrate intake correlated negatively with the Proteobacteria phylum (Supplemental Information R4).

\section{Discussion}

Bariatric surgery has been shown to reverse some of the deleterious effects to the multiple organ systems and metabolic pathways that are disrupted in T2D. However, the mechanisms behind the resolution of T2D are not well understood. It is thought that a complex interplay of weight dependent and independent factors ${ }^{14}$ are involved including: reduced adipose tissue leading to reduced inflammation and improved insulin sensitivity ${ }^{15}$; changes in the architecture of pancreatic islets such as increased beta cell mass ${ }^{16,17}$; changes in energy homeostasis and mitochondrial function ${ }^{18}$; restoration of bile acid levels and their impact on the farnesoid-X receptor (FXR) and the transmembrane $\mathrm{G}$ protein-coupled receptor 5 (TGR5) ${ }^{19}$, 20; enhanced release of gut hormones (e.g. $\mathrm{GLP}^{21}{ }^{2}$, ghrelin $\left.{ }^{22}\right)$; reversal of reduced enteroplasticity ${ }^{23}$; and via modulation of the gut microbiome, which is known to contribute to $\mathrm{BMI}$ and other risk factors for $\mathrm{T}^{2} \mathrm{D}^{24}$. In addition, bariatric surgery 
has been shown to normalise gene expression that is dysregulated in T2D, particularly mitochondrial genes and those involved with aerobic metabolism ${ }^{25}$. Here we show the contrasting impact of T2D and bariatric surgery on multiple pathways such as aromatic amino acid metabolism (tyrosine), branched chain amino acid metabolism (valine, leucine, isoleucine), one-carbon metabolism (methionine, sarcosine), short chain acyl carnitines (C3, C4, C5:1) and anaerobic glycolysis (lactate, pyruvate), which were higher in T2D and reduced following bariatric surgery. Similarly, other amino acids (glutamine, histidine), bile acids (GHCA, THCA, GUDCA) and lipids (HDL, acyl-alkylphosphatidylcholines C32:1, C38:5, C40:5, C42:5, C44:5, C44:6, Sphingomyelins C16:0, C18:0, C18:1, C20:2, C24:1, C26:1) were lower in T2D and increased following bariatric surgery.

The associations of these metabolites with BMI and glycaemic control are described below. However, although the differential metabolite set representative of bariatric surgery overlapped with both diabetes (19.3\% commonality) and BMI (18.6\% commonality), the percentage overlap between diabetes and BMI was minimal (4.0\% commonality). Thus, it appears that the change in metabolism with respect to resolution of $\mathrm{T} 2 \mathrm{D}$ is at least in part independent of $\mathrm{BMI}$ reduction, consistent with the observation that BMI and $\mathrm{HbA} 1 \mathrm{c}$ were differentially associated with metabolic profiles (Figures 1 \& 6).

As previously described ${ }^{26}$ the clinical and metabolic impact of RYGB was greater than VSG. The RYGB procedure was strongly associated with altered functionality of the gut microbiome as reflected in the urine, serum and faecal metabolomes and in the KEGG pathways associated with the altered microbiome. The microbiome associated with T2D was distinct from the characteristic non-diabetic microbiome but in general the perturbation caused by T2D was of a lower magnitude than the changes in microbial structure and function observed post bariatric surgery. The lesser extent of GM differences in T2D/non-T2D participants relative to the effects of bariatric surgery, coupled with the strong correlation of the GM to BMI but not HbA1c (Figure 6), suggests that the GM predominantly effects weight-dependent mechanisms. However, although the impact of T2D on the GM was more subtle, nevertheless we identified changes in several metabolite groups that correlated with GM changes after bariatric surgery. As discussed below, some of these metabolites, such as BCAAs, influence glycaemic control and may be important in weight-independent mechanisms of T2D resolution following bariatric surgery. 
413 Although we found differences in various individual taxa within the GM of T2D participants compared 414 to non-diabetic BMI matched controls, there was no overall difference detected in $\beta$-diversity. 415 Similarly, others have found only moderate differences in individuals with T2D and large inter416 individual variability ${ }^{1,27}$. Some of the differences in this cohort, such as lower Clostridium bartlettii 417 (aka Intestinibacter bartlettii) levels relative to non-diabetic controls, are in keeping with changes 418 due to metformin use (86\% metformin use in T2D group, see Supplemental Information R1) 28. 419 However, other differences in T2D participants such as lower Escherichia coli were apparent despite 420 metformin's well characterised action in increasing its relative abundance ${ }^{28,29}$. Escherichia 421 subsequently increased following both RYGB and VSG surgery.

422 In contrast, large metabolic dissimilarities occurred in T2D participants compared to non-diabetic 423 controls and we have defined a T2D metabolic signature, characterised by altered branched chain 424 and aromatic AA, one-carbon, acylcarnitine, lipid, BA and SCFA metabolism. The association of lysine 425 and 2-aminoadipate with $\mathrm{HbA1c}$ is consistent with the fact that both metabolites have been 426 associated with increased risk of T2D in the PREDIMED and Framingham Offspring Studies ${ }^{30,} 31$. 427 However, other studies seem to indicate that 2-aminoadipate can modulate insulin secretion and 428 reduce the impact of diabetes ${ }^{32}$. These contrasting results may be due to Maillard reactions which 429 are increased in diabetes and other age related diseases ${ }^{33}$. Similarly, inflammation has been shown 430 to promote the conversion of tryptophan to kynurenine and increased circulating kynurenine has 431 been previously associated with $\mathrm{HbA} 1 \mathrm{c}$ and diabetes ${ }^{34}$, 35. Likewise, methionine is susceptible to 432 oxidation to methionine sulfoxide, which was increased in serum in the T2D participants. This has 433 been associated with ageing and is consistent with higher oxidative stress in the T2D participants ${ }^{36}$.

\section{Multi-omic assessment of changes post RYGB and VSG surgery}

436 Reductions in the intake of all food groups, in particular carbohydrate, fat, sugar and fibre are 437 commonly noted after RYGB and VSG. In addition to the restrictive elements of both procedures 438 through a reduction in stomach size, bariatric procedures are known to reduce hunger and increase 439 satiety through modulation of anorexigenic hormones ${ }^{37}$. Patients also have a lower brain-hedonic 440 response to food ${ }^{38}$ and altered food preferences ${ }^{39}$. This change appears to be reflected here with an 441 increase in dietary healthiness, measured using the AHEI-2010 score, after surgery. However, it 
442 should be noted that all self-reported dietary data are subject to misreporting ${ }^{40}$. Although both 443 surgical procedures increased diet healthiness, several microbial and metabolic changes post-surgery 444 were distinct between interventions thereby implying that dietary change was not the main driver in 445 these effects. Nevertheless, a number of metabolic effects due to dietary changes were observed 446 and are discussed in the relevant sections below.

447 The magnitude of alteration in faecal bacterial composition following bariatric surgery was far greater 448 than the perturbation in the GM that was associated with T2D. This was also consistent with the 449 alteration in microbial metabolites identified in the serum, faeces, and particularly the urine 450 following bariatric surgery (hippurate, PAG, 4-cresyl sulfate, indoxyl sulfate and TMAO) whereas 451 these metabolites did not strongly differentiate non-diabetics from individuals with T2D. GM 452 composition changes along the intestine's length due to a gradient in a number of factors such as 453 nutrient availability, oxygen levels, $\mathrm{pH}$ and antimicrobial activity including BA levels ${ }^{41}$. The changes 454 to the GM after RYGB in this cohort represent a shift in the colonic bacteria towards those usually 455 found in higher concentrations in the small bowel ${ }^{42,43}$. Additionally, representation of a number of 456 obligate anaerobes increased. Whereas fermentative bacteria, usually found in high concentrations 457 in the colon, decreased after RYGB ${ }^{43}, 44$. As expected, due to the fact that VSG maintains continuity 458 of the gastrointestinal tract, the bacterial changes following VSG were more subtle. Nevertheless, 459 several species increased after VSG from genera that increased after RYGB. Initial studies 460 investigating obesity and the gut microbiota found a higher Firmicutes/Bacteroidetes ratio in obese 461 individuals ${ }^{2}, 45$, while other studies have reported contrasting results, finding the opposite changes or 462 no difference ${ }^{46,47}$. We found no significant change after either procedure, supporting the notion that 463 the picture is more complex than changes at a phylum level and that changes at lower taxonomic 464 levels and at a functional level may be more relevant ${ }^{48}$. Functional analysis of our cohort revealed a 465 significant shift towards increased bacterial proteolytic fermentation (putrefaction) pathways after 466 RYGB. This increase occurred despite a decrease in dietary protein consumption, although it is 467 possible that malabsorption resulting from altered small-intestine anatomy leads to higher $A A$ 468 concentrations reaching the colon. The change was corroborated by an increase in a number of 469 bacterially derived metabolites, generated through the fermentation of AAs. These included urinary 470 PAG, indoxylsulfate and 4-cresylsulfate derived from the bacterial metabolism of phenylalanine, 471 tryptophan and tyrosine respectively. Each of these amino acid - microbial metabolite pairs had a 472 significant inverse correlation, consistent with upregulated fermentation of proteins and AAs. 
473 Isovalerate and 2-methylbutyrate, derived from bacterial-degradation of BCAAs, were also increased 474 in faeces and urine after RYGB. Urinary PAG has previously been associated with a lean phenotype ${ }^{49}$ 475 and was found to be higher after RYGB in a rat model ${ }^{50}$. In this study, PAG was also correlated with a 476 healthier diet (lower carbohydrate and fat intake, including trans and polyunsaturated fats). Similarly, 477 the increase in hippurate, the glycine conjugate of benzoate, post-surgery is consistent with a lean 478 phenotype and increased fruit intake, as well as reduced risk of metabolic syndrome independent of $479 \operatorname{diet}^{51}$. Although bacterial pathways relating to proteolytic fermentation were not significantly 480 increased after VSG, a similar but less pronounced increase in related metabolites also occurred, 481 suggesting that these pathways were also functionally increased after VSG.

482 Perturbations in AA homeostasis, particularly BCAA and AAAs are associated with insulin resistance ${ }^{52}$ 483 and future risk of diabetes ${ }^{53}$. In this study we identified a higher Fischer ratio (BCAA/AAA), previously 484 associated with worsening liver function, in participants with T2D relative to non-diabetics ${ }^{54,55}$. BCAA 485 and AAAs both reduced following surgery, but the Fischer ratio only reduced following surgery in 486 participants with T2D and impaired glucose tolerance and was not significantly altered following 487 surgery in non-diabetics. The association of BCAA with diabetes is thought to be causative ${ }^{56}$. BCAA 488 infusions in rats and humans leads to the development of insulin resistance ${ }^{57}$. This led us to conclude 489 that the observed changes in AA profiles after surgery are likely to be an important factor driving 490 improved insulin resistance. Our findings suggest that the changes in AA profiles resulted in part from 491 increased AA putrefaction by the GM after RYGB. Consequently, RYGB may confer a metabolic 492 advantage for patients with T2D compared to VSG, through decreased availability and absorption of 493 BCAA from the gut. In support of this, germ-free mice have significantly altered profiles of AAs 494 absorbed from the gut via the portal vein relative to those with a normal $\mathrm{GM}^{58}$. This difference is due 495 to the large number of bacteria involved in AA biosynthesis and fermentation ${ }^{59,60}$. In a prior study, 496 oral gavage of mice with Bacteroides thetaiotaomicron led to a reduction in glutamate, 497 phenylalanine, leucine and valine ${ }^{10}$. Here two species of Paraprevotella, highly capable of producing 498 AAs, correlated with serum alanine levels. Perhaps more importantly we also identified a number of 499 negative correlations between bacterial species and serum AA levels, indicating that fermentation of 500 AAs by gut bacteria is an important pathway influencing human AA profiles. In particular, species 501 from the genera Streptococcus and Clostridium, important AA fermenters ${ }^{60}$, correlated with a 502 reduction in serum BCAAs (Supplemental Information R4). However, further studies are needed that 503 investigate the combined microbial, dietary and human contribution towards circulating levels of 
504 BCAAs. For example, we also note that the BCAA leucine was negatively correlated with dietary 505 healthiness (AHEI-2010 score) in this cohort.

506 In keeping with greater changes to the GM following RYGB, urinary TMAO was significantly increased 507 after RYGB but not VSG. Reduction of TMAO and other dietary nutrients such as choline and L508 carnitine to trimethylamine (TMA) is performed by the GM (predominantly Enterobacteriaceae). 509 Absorbed TMA is then converted back to TMAO by host hepatic enzymes ${ }^{61}$. TMAO has been described 510 as pro-atherogenic and high serum levels of TMAO have been proposed to be a predictor of 511 cardiovascular disease ${ }^{53,62}$. However, the causative effect of cardiovascular disease from high TMAO 512 is disputed and these results may have been due to confounders such as reduced kidney function 513 and poor metabolic control ${ }^{63}$. Other evidence such as the presence of high concentrations of TMAO 514 in elite athletes, as well as in the urine of Japanese populations whose diet contains a high portion of 515 fish and who have a low risk of cardiovascular disease, suggests that the causal role of TMAO in 516 cardiovascular disease is complex and conditional on a wealth of host-microbiota factors ${ }^{64,} 65$. 517 Moreover, TMAO was recently found to protect against impaired glucose tolerance and reduce 518 endoplasmic reticulum stress ${ }^{66}$.

519 The initial gateway step in biotransformation of primary conjugated BAs to secondary BAs is 520 performed by gut bacteria containing the bile salt hydrolase (BSH) enzyme. Interestingly, although 521 we observed increased secondary BAs, we found that the BSH gene load was decreased after RYGB 522 with no overall change after VSG. Furthermore, there were no significant changes in other BA 523 enzymes such as hydroxysteroid dehydrogenases. We concluded that the profound changes in the 524 BA pool composition observed after both procedures, including increased secondary BAs, result 525 predominantly from changes in host factors such as altered hepatic processing and BA re-absorption. 526 In addition, there was a consistent increase in glycine conjugation of BAs relative to taurine in serum 527 after RYGB. Changes in glycine/taurine conjugation can result from changes in their bioavailability in 528 the liver ${ }^{67}$ and certainly there was increased serum glycine in this cohort. In addition, bacterial glycine 529 metabolism pathways were increased after RYGB and this may also influence the bioavailability of 530 glycine for conjugation. Indeed, germ-free animals excrete almost exclusively tauro-conjugated $531 \mathrm{BAs}^{68}$. Interestingly, GHCA had a strong negative correlation with HbA1c in this cohort. Diabetes has 532 been associated with lower serum concentrations of HCA species and they are strong predictors of 533 metabolic disease ${ }^{69}$. Administration of hyocholic acid by others increased serum fasting GLP-1 in 534 healthy and diabetic mouse models by simultaneously activating TGR5 and inhibiting FXR, a unique 
535 mechanism not found in other BA species ${ }^{70}$. However, the overall metabolic effects of differences in 536 the BA pool and conjugation patterns are difficult to predict ${ }^{20}$.

537 SCFAs are produced through the bacterial fermentation of dietary fibre and complex carbohydrates.

538 Faecal acetate decreased after both procedures, likely due to a significant reduction in dietary 539 substrate. Indeed, faecal acetate was negatively correlated with dietary carbohydrate and calorie 540 intake. However, the reduction in butyrate and valerate seen after VSG was not replicated after 541 RYGB, despite a similar reduction in dietary substrate. This discrepancy suggests that the GM 542 following RYGB is able to produce these SCFA more readily than the microbiota after VSG. The SCFA 543 signature is important as each SCFA has a unique impact on the host. Acetate production leads to a 544 positive feedback loop that increases appetite, induces lipid deposition in liver and skeletal muscle, 545 and increases insulin resistance ${ }^{71}$. Whereas butyrate has a beneficial role in host satiety, insulin 546 resistance and colonocyte health ${ }^{72}$.

547 Crucially, although the overall changes after bariatric surgery appear to be towards a healthier 548 phenotype there may be some negative consequences of surgery. Increased protein metabolism 549 within the gut is usually considered to be harmful ${ }^{73}$. Putrefaction results in the production of toxic 550 compounds such as amines, sulphides and ammonia ${ }^{74}$, whilst phenols and indoles, increased after 551 bariatric surgery in this cohort, are reported to be pro-inflammatory and cytotoxic ${ }^{75,76}$.

552 In conclusion, metabolic changes post-surgery were achieved by both weight-dependent and weight553 independent processes and we have identified multi-omic signatures specific to obese and T2D states 554 at the systems level, some of which demonstrated contrasting patters when compared with the 555 effects of bariatric surgery. BMI correlated inversely with bacterially derived urinary metabolites such 556 as PAG, 4-cresylsulfate and indoxylsulfate and positively with faecal acetate and valerate, whereas $557 \mathrm{HbA1C}$ showed stronger correlation with serum AAs, acylcarnitines, kynurenine and 2-aminoadipate. 558 Greater functional and taxonomic changes were observed in the GM following RYGB compared to 559 VSG. These microbial changes, particularly after RYGB, appeared to influence the complex 560 relationship between the GM and host metabolism. The abundance of amino acid metabolism 561 pathways within the GM and corresponding metabolites of protein putrefaction increased after 562 RYGB, despite reduced dietary protein intake, and correlated with decreased serum BCAAs. 563 Ultimately, further mechanistic work is needed to better understand these GM-host co-metabolism 564 pathways and to establish their full effects, including those that may be harmful to health. 


\section{Methods}

\section{Resource availability}

\section{Lead contact}

568 Further information and requests for resources and reagents should be directed to and will be 569 fulfilled by the Lead Contact, Elaine Holmes (elaine.holmes@imperial.ac.uk).

570 Materials availability

571 This study did not generate new unique reagents.

572 Data and code availability

573 Metagenomic data has been deposited with GenBank, EMBL and DDBJ databases under the 574 BioProject accession number PRJNA473348.

575 Further metagenomic and metabolic data have been deposited at Mendeley Data: 576 http://dx.doi.org/10.17632/t76nm3yfzh.1

\section{Experimental model and participant details}

\section{Recruitment}

579 Patients referred for consideration of bariatric surgery who were obese $\left(\mathrm{BMI}>30 \mathrm{~kg} / \mathrm{m}^{2}\right)$, aged $\geq 18$, 580 had failed efforts at lifestyle modification and dieting and were willing to comply with the trial 581 protocol were recruited prospectively. Diabetics ( $\mathrm{HbA1c}>48 \mathrm{mmol} / \mathrm{mol}$ or treated) and non-diabetics 582 were eligible for recruitment.

583 Patients who had previously undergone bariatric or major abdominal surgery, were or intended to 584 become pregnant during trial period, or took long-term antibiotics were excluded. Major abdominal 585 surgery included patients who had undergone small or large bowel resection, liver, pancreatic, 586 splenic or stomach surgery, as these could influence the gut microbiota and / or the patient's 587 metabolic state. Patients that had previously had an appendicectomy, cholecystectomy or hernia 588 repair were not excluded. 
589 The study protocol and sample collection instructions were co-developed with patient 590 representatives to help reduce the study burden for patients. To improve patient compliance sample 591 collection occurred at the time of patients' usual NHS appointments preoperatively and at 3-months 592 post-procedure. A small exploratory cohort were also sampled at 1-year post-procedure and are 593 reported in Supplemental Information.

\section{Metabolic surgery}

595 Participants underwent Roux-en-Y Gastric Bypass (RYGB) or Vertical Sleeve Gastrectomy (VSG) 596 surgery at a National Health Service (NHS) University Teaching Hospital in London, UK. A single dose 597 of $1.2 \mathrm{~g}$ intravenous co-amoxiclav was given during induction of anaesthesia (clindamycin if penicillin 598 allergic).

\section{Regulatory approvals}

600 The study received NHS Research Ethics Committee (15/ES/0026) approval and was registered with 601 ClinicalTrials.gov (NCT02421055).

602

603 Participants were assessed at the above time points for: 1) anthropometric \& physiological 604 measurements, 2) demographic details, 3) biochemical parameters including glycated haemoglobin 605 (HbA1c), 4) oral-hypoglycaemic, insulin and other medication use, length of diabetes diagnosis and 606 other co-morbidities.

608 An online self-reported $24 \mathrm{hr}$ dietary recall questionnaire (www.myfood24.org) was utilised to 609 capture detailed dietary intake information from patients at each of the study time points so that changes in diet following surgery could be accounted for in the analysis. Three $24 \mathrm{hr}$ recall questionnaires were completed at each time point. Participants were able to pick from a selection of pictures of corresponding foods to accurately ascertain portion quantities. Collected dietary information was used to calculate Alternative Healthy Eating Index 2010 (AHEI-2010) scores as described previously ${ }^{13}$. In brief, scores of 0-10 were given for 11 components (maximum score 110). High scores were given for a high intake of vegetables, fruit, nuts and legumes, whole grains, long chain omega-3 fats and polyunsaturated fats, moderate intake of alcohol and low intake of sugar, sweetened drinks and fruit juice, red and processed meat, trans-fat and sodium. 
619 Serum $(n=158)$, faecal $(n=81)$ and $24 \mathrm{hr}$ urine $(n=83)$ samples were collected preoperatively and at 3620 months (serum=49, faecal=27, urine=30) and 1-year postoperatively (exploratory 1-year data 621 reported in Supplemental Information due to low numbers), in a non-fasted state. 24-hour urine 622 samples were collected in sterile containers from 9am until 9am on the day of the study visit. Stool 623 samples were collected using a Faecotainer ${ }^{\circledR}$ collection kit and stored on an ice pack provided, as 624 close as possible to but not more than 6 hours before the study visit. Serum was collected using red 625 top BD Vacutainer ${ }^{\circledR}$ serum tubes (no additive) and processed according to manufacturer guidelines. 626 After collection, samples were aliquoted and stored at $-80^{\circ} \mathrm{C}$ until analysis. Prior to freezing, a 627 separate aliquot of homogenised stool was used to generate faecal water as follows: approximately $62810 \mathrm{~g}$ of stool was added to 4 parts $\mathrm{HPLC}$ grade $\mathrm{H}_{2} \mathrm{O}(\mathrm{g} / \mathrm{ml})$, vortexed at $2850 \mathrm{rpm}$ for $15 \mathrm{~min}$ then 629 centrifuged at $10000 \mathrm{~g}$ for $15 \mathrm{~min}$ at $4^{\circ} \mathrm{C}$. The resulting supernatant (faecal water) was frozen at $-80^{\circ} \mathrm{C}$ 630 until analysis ${ }^{77}$.

631 Method details

${ }^{1} \mathrm{H}-\mathrm{NMR}$ metabolite analysis

\section{Sample preparation}

634 Urine and faecal water samples were prepared for analysis by ${ }^{1} \mathrm{H}-\mathrm{NMR}$ spectroscopy as follows: 635 frozen samples $\left(-80^{\circ} \mathrm{C}\right)$ were thawed, vortexed and then centrifuged at $1600 \mathrm{~g}$ for $10 \mathrm{~min}$ to remove 636 particulates and precipitated proteins. Faecal water supernatant was further filtered through Micro 637 centrifuge filters $\left(0.45 \mu \mathrm{m}\right.$ Nylon, Costar) at $16000 \mathrm{~g}$ for $15 \mathrm{~min}$ at $4^{\circ} \mathrm{C} .540 \mu \mathrm{L}$ of each sample was mixed

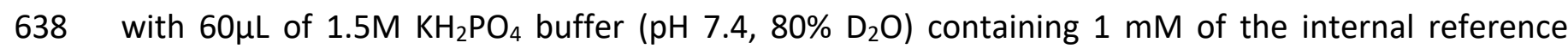
639 standard, 3-(trimethylsilyl)-[2,2,3,3,-2 $\left.\mathrm{H}_{4}\right]$-propionic acid (TSP) and $2 \mathrm{mM}$ sodium azide $\left(\mathrm{NaN}_{3}\right)$, as 640 described previously ${ }^{78}$.

641 After thawing, serum samples were centrifuged at $12000 \mathrm{~g}$ for 5 minutes at $4^{\circ} \mathrm{C}$. Subsequently, $300 \mu \mathrm{L}$ 642 of serum was mixed with $300 \mu \mathrm{L}$ of $0.075 \mathrm{M} \mathrm{NaH}_{2} \mathrm{PO}_{4}$ buffer $(\mathrm{pH} 7.4)$ containing $0.8 \mathrm{mM}$ of the internal 643 reference standard, 3-(trimethylsilyl)-[2,2,3,3,-2 $\left.\mathrm{H}_{4}\right]$-propionic acid (TSP) and 3.1mM sodium azide $644\left(\mathrm{NaN}_{3}\right)$, as described previously ${ }^{78}$. 
$646{ }^{1} \mathrm{H}-\mathrm{NMR}$ spectroscopy was performed at $300 \mathrm{~K}$ on Bruker $600 \mathrm{MHz}$ (urine and serum) and $800 \mathrm{MHz}$ 647 (faecal water) spectrometers (Bruker Biospin) using the following standard one-dimensional pulse 648 sequence: $R D-g_{z 1}-90^{\circ}-t_{1}-90^{\circ}-t_{m}-g_{z 2}-90^{\circ}-A C Q^{78}$. The relaxation delay (RD) was set at $4 \mathrm{~s}$, $64990^{\circ}$ represents the applied $90^{\circ}$ radio frequency pulse, interpulse delay $\left(\mathrm{t}_{1}\right)$ was set to an interval of $6504 \mu \mathrm{s}$, mixing time $(\mathrm{tm})$ was $10 \mathrm{~ms}$, magnetic field gradients $\left(\mathrm{g}_{\mathrm{z} 1}\right.$ and $\left.\mathrm{g}_{\mathrm{z} 2}\right)$ were applied for $1 \mathrm{~ms}$ and the 651 acquisition period (AQA) was 2.7s. Water suppression was achieved through irradiation of the water 652 signal during RD and $t_{m}$. For the urine samples, each spectrum was acquired using 4 dummy scans 653 followed by 32 scans while faecal spectra were acquired using 256 scans and 4 dummy scans and 654 collected into $64 \mathrm{~K}$ data points. A spectral width of $12,000 \mathrm{~Hz}$ was used for all the samples. Prior to 655 Fourier transformation, the free induction decays (FIDs) were multiplied by an exponential function 656 corresponding to a line broadening of $0.3 \mathrm{~Hz}$. Serum samples were analysed by ${ }^{1} \mathrm{H}-\mathrm{NMR}$ using the 657 standard one-dimensional pulse sequence described above and Carr-Purcell-Meiboom-Gill (CPMG) 658 one dimensional pulse sequences. CPMG was used to attenuate broad, interfering peaks from lipids 659 and proteins present in serum. The CPMG pulse sequence had the form RD- $90^{\circ}-\left(t-180^{\circ}-t\right) n-A C Q$. The 660 acquisition parameters were set using the same settings as the standard 1D pulse sequence, with the 661 spin-echo delay (t) set at $0.3 \mathrm{~ms}$ and 128 loops ( $n$ ) performed. Continuous wave irradiation was 662 applied at the water resonance frequency during the relaxation delay (RD).

Pre-processing

$664{ }^{1} \mathrm{H}-N M R$ spectra were automatically corrected for phase and baseline distortions and referenced to 665 the TSP singlet at $\delta 0.0$ using TopSpin 3.1 software. Spectra were then digitized into 20K data points 666 at a resolution of $0.0005 \mathrm{ppm}$ using an in-house MATLAB R2014a (Mathworks) script. Subsequently, 667 spectral regions corresponding to the internal standard ( $\delta-0.5$ to 0.5$)$ and water ( $\delta 4.6$ to 5$)$ peaks 668 were removed. In addition, urea ( $\delta 5.4$ to 6.3 ) was removed from the urinary and serum spectra due 669 to its tendency to cross-saturate with the suppressed water resonance. All spectra were normalised 670 using median fold change normalisation using the median spectrum as the reference ${ }^{79}$. 
673 Quantitative analysis of 57 bile acids was performed using an established technique ${ }^{80}$. The method

674 was adapted for analysis of bile acids in faecal samples.

675 Sample preparation

676 Bile acids were extracted from serum using the following method: $100 \mu \mathrm{L}$ of serum was vortexed with

$677280 \mu \mathrm{L}$ of $\mathrm{MeOH}$. Samples were centrifuged at $14000 \mathrm{~g}$ for $15 \mathrm{~min}$ at $4^{\circ} \mathrm{C}$, followed by incubation at $67820^{\circ} \mathrm{C}$ for $20 \mathrm{~min}$. Internal standards (16 deuterated bile acids) were added to the supernatant at a final 679 concentration of 50nM.

680 Bile acids were extracted from faecal samples using the following method. Faecal samples were first 681 freeze-dried. 100mg of freeze-dried material was then placed in microtubes with 1ml of 2:1:1 $\mathrm{H}_{2} \mathrm{O}$ : 682 Acetonitrile (ACN): Isopropanol (IPA) and approximately 50mg of $1 \mathrm{~mm}$ Zirconia beads. This 683 underwent $3 \times 30$ seconds bead beating and a Biospec bead beater followed by centrifugation at $68416,000 \mathrm{~g}$ for $20 \mathrm{~min}$ at $4^{\circ} \mathrm{C}$. The supernatant was further filtered through Micro-centrifuge filters $685\left(0.45 \mu \mathrm{m}\right.$ Nylon, Costar) at $16000 \mathrm{~g}$ for $15 \mathrm{~min}$ at $4^{\circ} \mathrm{C}$. To ensure bile acid concentrations were within 686 the dynamic range of the machine extracts were diluted 1:25 and 1:200 prior to analysis using the $687 \mathrm{H}_{2} \mathrm{O}: \mathrm{ACN}$ :IPA mix. Internal standard (16 deuterated bile acids) was added to the filtered supernatant 688 at a final concentration of 50nM.

\section{LC-MS machine conditions}

690 BA analysis was performed using an ACQUITY ultra-performance liquid chromatography (UPLC) 691 coupled to a Xevo triple quadrupole (TQ-S) mass spectrometer.

692 For liquid chromatography, an ACQUITY BEH C8 column $(1.7 \mu \mathrm{m}, 100 \mathrm{~mm} \times 2.1 \mathrm{~mm})$ was used at an 693 operating temperature of $60^{\circ} \mathrm{C}$. The mobile phase solvent $A$ consisted of a $1: 10 \mathrm{ACN}: \mathrm{H}_{2} \mathrm{O}$, with $1 \mathrm{mM}$ 694 ammonium acetate and pH 4.15 adjusted with acetic acid. Mobile phase solvent B consisted of 1:1 695 ACN:IPA. The chromatographic gradient was as previously published ${ }^{80}$.

696 Mass spectrometry was performed in negative ionisation mode (ESI-) using the following parameters: 697 capillary voltage $1.5 \mathrm{kV}$, cone voltage $60 \mathrm{~V}$, source temperature $150^{\circ} \mathrm{C}$, desolvation temperature $698600^{\circ} \mathrm{C}$, desolvation gas flow $1000 \mathrm{~L} / \mathrm{hr}$, and cone gas flow 150L/hr. 57 bile acid species (36 non699 conjugated, 12 taurine conjugated, 9 glycine conjugated) were assayed using multiple reaction 
700 monitoring (MRM). The transitions for each bile acid and deuterated internal standard was as 701 previously published ${ }^{80}$.

702

703 Quantitative analysis of SCFAs and other Carboxylic Acids

704 A total of five short/medium chain fatty acids, three methyl-branched SCFAs and two hydroxyl 705 carboxylic acids were analysed by GC-MS using a method adapted from Moreau et al ${ }^{81}$.

707 After defrosting and mixing, $100 \mu \mathrm{L}$ of urine / serum was aliquoted with $500 \mu \mathrm{L}$ of methyl tert-butyl 708 ether (MTBE) with 100ppm of internal standard (methyl stearate) and $2 \mu \mathrm{L}$ of HCL. This was vortexed 709 and then shaken for $20 \mathrm{~min}$. Following this samples were centrifuged at $10000 \mathrm{~g}$ for $5 \mathrm{~min}$ at $4^{\circ} \mathrm{C}$. Next, $71090 \mu \mathrm{L}$ of the polar phase was placed into a silanised vial and vortexed with $150 \mu \mathrm{L}$ of derivatiser $N$-tert711 butyldimethylsilyl- $N$-methyltrifluoroacetamide with $1 \%$ tert-butyldimethylchlorosilane (MTBSTFA + $7121 \% \mathrm{TBDMSCI}$ ). This was then incubated for $45 \mathrm{~min}$ at $60^{\circ} \mathrm{C}$ before aliquoting into silanised inserts for 713 analysis.

714 The method was modified to account for higher levels of SCFA in stool. After defrosting, 100mg of 715 stool was aliquoted with $1,000 \mu \mathrm{L}$ of MTBE with $100 \mathrm{ppm}$ of internal standard (methyl stearate) and $7164 \mu \mathrm{L}$ of $\mathrm{HCL}$. $30 \mu \mathrm{L}$ of the polar phase was mixed with $150 \mu \mathrm{L}$ of derivatiser.

\section{GC-MS machine conditions}

718 Derivatised samples were analysed by GC-MS with a Bruker triple quadrupole (TQ) GC-MS/MS. 719 Helium was used as a carrier gas at a constant flow rate of $1.5 \mathrm{ml} / \mathrm{min}$ through the column. The 720 injector temperature was $250^{\circ} \mathrm{C}$ with a split ratio $1: 10$. The temperature of the oven was started at $72140^{\circ} \mathrm{C}$ and increased at the rate of $46^{\circ} \mathrm{C} / \mathrm{min}$ to $127^{\circ} \mathrm{C}, 2^{\circ} \mathrm{C} / \mathrm{min}$ to reach $131^{\circ} \mathrm{C}, 30^{\circ} \mathrm{C} / \mathrm{min}$ to reach $722160^{\circ} \mathrm{C}$, then $50^{\circ} \mathrm{C} / \mathrm{min}$ to reach a final temperature of $300^{\circ} \mathrm{C}$. The transfer line to the mass 723 spectrometer was set at $280^{\circ} \mathrm{C}$. Targeted analysis of the ten compounds and internal standard was 724 performed in multiple reaction monitoring mode (MRN) using the following settings:

\begin{tabular}{|l|l|l|l|}
\hline Compound & Quantifier $(\mathbf{m} / \mathbf{z})$ & Qualifier $(\mathbf{m} / \mathbf{z})$ & Collision Energy $(\mathbf{e V})$ \\
\hline Acetate & $117 \rightarrow 75$ & $117 \rightarrow 47$ & 10 \\
\hline Propionate & $131 \rightarrow 75$ & $131 \rightarrow 47$ & 10 \\
\hline
\end{tabular}




\begin{tabular}{|l|l|l|l|}
\hline Butyrate / Isobutyrate & $145 \rightarrow 75$ & $145 \rightarrow 43$ & 10 \\
\hline Valerate / Isovalerate & $159 \rightarrow 75$ & $159 \rightarrow 57$ & 12 \\
\hline 2 Methylbutyrate & $159 \rightarrow 75$ & $159 \rightarrow 57$ & 12 \\
\hline 2 Hydroxybutyrate & $147 \rightarrow 73$ & $147 \rightarrow 45$ & 20 \\
\hline Caproate & $173 \rightarrow 75$ & $173 \rightarrow 81$ & 15 \\
\hline Lactate & $147 \rightarrow 73$ & $147 \rightarrow 45$ & 20 \\
\hline Methyl stearate (IS) & $87 \rightarrow 55$ & $87 \rightarrow 59$ & 10 \\
\hline
\end{tabular}

\section{Quantitative serum metabolite analysis}

727 Quantitative analysis of other metabolites in serum samples, including amino acids, biogenic amines, 728 acylcarnitines, phosphatidylcholines, lysophosphatidylcholines and sphingolipids was performed 729 using the Biocrates AbsoluteIDQ ${ }^{\circledR}$ p180 kit, according to the manufacturer guidelines ${ }^{82}$. Samples were 730 analysed using flow injection analysis (FIA)-MS/MS and LC-MS/MS for different metabolite groups.

\section{Sample preparation}

$73210 \mu \mathrm{L}$ of serum sample / PBS / calibration / QC and 10 $\mu \mathrm{L}$ of the ISTD mix (except in blanks) was added 733 to each well. This was dried for $30 \mathrm{~min}$ under nitrogen flow. Following this, $50 \mu \mathrm{L}$ of the derivatization 734 solution was pipetted into each well. The plate was covered and incubated for $20 \mathrm{~min}$, then dried for $73560 \mathrm{~min}$ under nitrogen flow. Next, $300 \mu \mathrm{L}$ of extraction solvent was added to each well, shaken for $73630 \mathrm{~min}$ at $450 \mathrm{rpm}$, then centrifuged for $2 \mathrm{~min}$ at $500 \mathrm{~g}$. For the LC-MS/MS $150 \mu \mathrm{L}$ was added to $150 \mu \mathrm{l}$ $737 \mathrm{H}_{2} \mathrm{O}$. For the FIA, $15 \mu \mathrm{L}$ was added to $750 \mu \mathrm{L}$ of FIA mobile phase. Both plates were shaken for $2 \mathrm{~min}$ at $738600 \mathrm{rpm}$.

\section{Machine conditions}

740 Samples were analysed using a Waters I-Class UHPLC system and Waters Xevo TQ-S tandem mass 741 spectrometer.

742 For FIA-MS/MS (direct infusion): the FIA mobile phase consisted of Biocrates Solvent I $+290 \mathrm{~mL}$ $743 \mathrm{MeOH}$. A $2 \mathrm{~min}$ isocratic method was used, starting at $0.15 \mathrm{~mL} / \mathrm{min}$ for $0.1 \mathrm{~min}$, gradually decreasing 744 to $0.03 \mathrm{~mL} / \mathrm{min}$ at $1 \mathrm{~min}$, increasing to $0.2 \mathrm{~mL} / \mathrm{min}$ at $1.5 \mathrm{~min}$, to $0.8 \mathrm{~mL} / \mathrm{min}$ at $1.60 \mathrm{~min}$, and finally 745 decreasing to $0.15 \mathrm{~mL} / \mathrm{min}$ at $1.95 \mathrm{~min}$. MS settings were: capillary voltage $3.2 \mathrm{kV}$, cone voltage $10 \mathrm{~V}$, 
source offset $50 \mathrm{~V}$, source temp $150^{\circ} \mathrm{C}$, desolvation temp $620^{\circ} \mathrm{C}$, cone gas $150 \mathrm{~L} / \mathrm{H}$, desolvation gas $1000 \mathrm{~L} / \mathrm{H}$, collision gas flow $0.15 \mathrm{~mL} / \mathrm{min}$, probe position $5 \mathrm{~mm}$.

748 For LC-MS/MS: A Waters Acquity UPLC BEHC18 1.7 $\mu \mathrm{m} 2.1 \times 75 \mathrm{~mm}$ column was used. Mobile phase 749 A: $1000 m L ~ H_{2} \mathrm{O}+2 \mathrm{~mL}$ formic acid (FA), Mobile phase B: $500 \mathrm{~mL} A C N+1 \mathrm{~mL} F$. Gradient elution was 750 used; starting at a flow rate of $0.8 \mathrm{~mL} / \mathrm{min}$ with $100 \% \mathrm{~A}$ for $0.45 \mathrm{~min}$, then changing in a linear gradient 751 to $85 \% \mathrm{~A}$ at $3.3 \mathrm{~min}$, to $30 \% \mathrm{~A}$ at $5.9 \mathrm{~min}$, to $100 \% \mathrm{~B}$ at $6.05 \mathrm{~min}$, flow then increased in a concave 752 gradient to $0.9 \mathrm{~mL} / \mathrm{min} 100 \%$ B by $6.20 \mathrm{~min}$, remaining at $0.9 \mathrm{~mL} / \mathrm{min} 100 \% \mathrm{~B}$ until $6.42 \mathrm{~min}$, before 753 decreasing back in a concave gradient to $0.8 \mathrm{~mL} / \mathrm{min} 100 \% \mathrm{~B}$ at $6.52 \mathrm{~min}$. The mobile phase was then 754 changed in a concave gradient from 100\% B to $100 \%$ A between 6.52 and $6.7 \mathrm{~min}$ and remained at $755100 \%$ A $0.8 \mathrm{ml} / \mathrm{min}$ until 7.3min. MS settings were: capillary voltage $3.9 \mathrm{kV}$, cone voltage $20 \mathrm{~V}$, source 756 offset $50 \mathrm{~V}$, source temp $150^{\circ} \mathrm{C}$, desolvation temp $350^{\circ} \mathrm{C}$, cone gas $150 \mathrm{~L} / \mathrm{Hr}$, desolvation Gas $650 \mathrm{~L} / \mathrm{Hr}$, 757 collision gas flow $0.15 \mathrm{~mL} / \mathrm{min}$, probe position $7 \mathrm{~mm}$.

758 Data were processed using targetlynx (Waters) and METIDQ (Biocrates; version Carbon) then 759 exported as a CSV file for statistical analysis.

761 Faecal metagenomic analysis

\section{DNA extraction}

763 Faecal samples were stored at $-80^{\circ} \mathrm{C}$ prior to analysis. DNA was extracted using the MoBio 764 PowerFaecal ${ }^{\circledR}$ DNA Isolation Kit, according to manufacturer's instructions. In brief, DNA was 765 extracted from two separate $0.25 \mathrm{~g}$ aliquots of mixed whole stool samples at each analysis point. 766 Samples were homogenised in $2 \mathrm{ml}$ bead beating tubes containing garnet beads. Cell lysis of host and 767 microbial cells was facilitated through both mechanical collisions between beads and chemical 768 disruption of cell membranes. The reagent to precipitate non-DNA organic and inorganic material 769 was then applied. Lastly, DNA was captured on a silica spin column, washed and eluted for downstream analysis. Quality control of DNA quality and quantity was assessed using an Agilent 4200 TapeStation.

773 Shotgun sequencing was performed using an Illumina HiSeq 4000 with paired-end 150bp reads. 774 Library preparation was undertaken using the NEBNext Ultra II DNA Library Prep Kit. 15 dual index 
775 barcodes (unique at both ends) were custom-designed and ordered from Integrate DNA Technologies $776\left(\right.$ (IDT $\left.^{\circledR}\right)$. Quality control of prepped libraries was performed using the Promega GloMax $^{\circledR}$ and 777 QuantiFluor ${ }^{\circledast}$ dsDNA systems. Each of the "uniquely dual-indexed" libraries were pooled and run on 778 a single lane of the HiSeq4000. A mean of $6.89 \mathrm{~Gb}$ sequence data was acquired for each of 120 779 samples (median 6.84, range $3.88-14 \mathrm{~Gb}$ ).

780 Processing of sequence data

781 There are known lane-swapping issues with the HiSeq 4000, leading to duplication of some 782 sequencing reads. For this reason, fastq files for each sample were subject to de-duplication using 783 FastUniq $^{83}$. Sequencing data were then processed using the Scalable Metagenomics Pipeline 784 (ScaMP) ${ }^{84}$, (http://www.imperial.ac.uk/bioinformatics-data-science-group/resources/software/ 785 scamp). In brief, raw sequence data were assessed for the presence of adapter sequences and 786 trimmed using Trim Galore! (Babraham Bioinformatics) to remove low-quality bases $(\mathrm{Q}<20)$ from 787 the $3^{\prime}$ end of reads and discard trimmed reads shorter than 100 nt. Quality control of trimmed reads 788 was performed using FastQC (Babraham Bioinformatics). Reads that mapped with BWA-MEM ${ }^{85}$ to 789 human genome (hg19) were removed from read pairs, as ethical permission is not available for use 790 of human data derived from metagenomes. Remaining reads were assumed to be microbial (bacteria, 791 archaea, virus, fungi, protozoa) and processed further. Trimmed sequence data with human reads 792 removed have been deposited with GenBank, EMBL and DDBJ databases under the BioProject 793 accession number PRJNA473348.

794 MetaPhIAn 2.686, 87 was used to determine the bacterial and archaeal taxonomic 795 composition/abundance for each sample. Metagenome assembly was carried out in two rounds 796 using metaSPAdes 3.11.088, with an initial independent assembly carried out for each sample. 797 Unassembled reads were then pooled and subjected to a second round of assembly to improve the 798 representation of low-abundance sequences. Taxa were normalised to relative abundance for 799 downstream analyses.

800 Ab-initio gene prediction was carried out using MetaGeneMark ${ }^{89,90}$. The resulting predictions were 801 translated, and the protein sequences clustered using the cluster-fast method of UCLUST ${ }^{91}$, with a 95 802 \% identity cut-off. Centroid sequences from each cluster were used to form a non-redundant gene 803 catalogue used for downstream analyses. Gene abundance in each sample was determined by 804 alignment of the reads using BWA-MEM against the gene catalogue, determining the number of 
reads mapped to each gene sequence and normalising as described ${ }^{92}$. Functional annotation to KEGG pathways was carried out by mapping centroid sequences to the eggNOG-Mapper database ${ }^{93}$

807 (version 4.5, downloaded on 1 March 2018) using diamond on our in-house server.

808 Microbial gene richness (MGR) was determined as described previously ${ }^{92,}$ 94. Briefly, data were 809 downsized to adjust for sequencing depth and technical variability by randomly selecting 7 million 810 reads mapped to the gene catalogue (of 11,005,136 genes) for each sample and then computing the 811 mean number of genes drawn over 30 random samplings.

\section{Quantification and statistical analysis}

\section{Pre-processing}

814 To correct for dilution differences between samples normalisation procedures were applied. Global 815 metabolite $\left({ }^{1} \mathrm{H}-\mathrm{NMR}\right.$ spectra) data sets were corrected for dilution effects using median fold change 816 normalisation ${ }^{79}$. Scaling to unit variance was then applied to serum and urine data sets, while pareto 817 scaling was used for faecal datasets, due to the presence of dominant and variable oligosaccharide 818 resonances. Targeted metabolites measured within urine samples were corrected for dilutional 819 differences using osmolality and creatinine measurements ${ }^{95}$. Metagenome data were expressed as 820 relative abundance. Taxa with low abundance (present in $<30 \%$ of both subgroups) were excluded 821 from downstream statistical analyses.

\section{Univariate analysis}

823 Due to the non-parametric nature of the results, differences between paired samples pre/post 824 intervention in quantified metabolites and within the gut microbiota were assessed for significance 825 using the Wilcoxon Rank test (two-sided). Differences in non-paired data were assessed using the 826 Mann-Whitney $U$ test (two-sided). P-values were adjusted for multiple testing using the Benjamini827 Hochberg (BH) False Discovery Rate method (pFDR). Phylogenetic Trees were generated to illustrate 828 significant gut microbiota changes using the GraPhIAn ${ }^{96}$ script in Python.

\section{Multivariate analysis}

830 Multivariate statistical analysis of normalised ${ }^{1} \mathrm{H}-\mathrm{NMR}$ spectra was performed using SIMCA 15 831 (Umetrics) ${ }^{79}$. Principal component analysis (PCA) was used to provide an overview of the data. 
832 Orthogonal Partial Least Squares - Discriminant Analysis (OPLS-DA) models were established based

833 on one predictive component and one orthogonal component to discriminate between samples from

834 T2D and non-diabetic participants. Unit variance scaling was applied to ${ }^{1} \mathrm{H}-\mathrm{NMR}$ spectral data. The fit 835 and predictability of the models obtained were determined by the $R^{2} Y$ and $Q^{2} Y$ values respectively. 836 Significant metabolites differentiating between groups were obtained from ${ }^{1} \mathrm{H}-\mathrm{NMR}$ OPLS-DA models 837 after investigating ${ }^{1} \mathrm{H}-\mathrm{NMR}$ signals with correlation coefficient values higher than 0.35. Jack-knifed $83895 \%$ confidence intervals of the coefficients were used to confirm significance of the variables.

839 Longitudinally paired global metabolic data, pre- and post-intervention, were analysed using 840 Repeated Measures, Monte-Carlo Cross-Validation, PLS-DA (RM-MCCV-PLSDA) ${ }^{97,} 98$ using covariate 841 adjusted projection to latent structures in MATLAB. Data were centred and scaled to account for the 842 repeated-measures design. 1000 MCCV models were generated and used to calculate the mean 843 cross-validated predictive component score $\left(T_{\text {pred }}\right)$ and variance for each sample ${ }^{98}$. The fit and 844 predictability of the models obtained was determined by the $R^{2} X$ and $Q^{2} Y$ values respectively. 845 Gaussian kernel density estimates of the $\mathrm{T}_{\text {pred }}$ in each group were generated for visual 846 interpretation ${ }^{98} .25$ bootstrap resamplings in each of the 1000 models was used to estimate the 847 variance and mean coefficient for each variable and derive a $p$ value for each variable accordingly ${ }^{98}$. 848 Benjamini-Hochberg false discovery corrections were performed and a variable was considered 849 significant with a false discovery rate value $(q) \leq 0.01$. Manhattan plots showing $-\log _{10}(q) \times$ sign of the 850 variable regression coefficient for each variable within each RM-MCCV-PLSDA model were 851 generated, with dotted lines added to illustrate the q value significance cut off level on the $\log _{10}$ scale.

852 Exploration of gut microbiota taxa was performed using Principal coordinates analysis (PCoA) of Bray853 Curtis dissimilarity matrices ( $\beta$-diversity) using the Vegan ${ }^{99}$ function in R. Significance of group 854 separation in $\beta$-diversity was assessed by permutational multivariate analysis of variance 855 (PERMANOVA). Nested PERMANOVA was used longitudinal analyses to account for the repeated 856 measures design.

\section{Metabolite identification}

858 A combination of data-driven strategies such as such as SubseT Optimization by Reference Matching 859 (STORM) $)^{100}$ and Statistical TOtal Correlation SpectroscopY (STOCSY) ${ }^{101}$ and analytical identification 860 strategies were used to aid structural identification of significant discriminatory metabolites. 861 Specifically, a catalogue of $1 \mathrm{D}^{1} \mathrm{H}-\mathrm{NMR}$ sequence with water pre-saturation and 2D NMR experiments 
862 such as J-Resolved spectroscopy, ${ }^{1} \mathrm{H}-{ }^{1} \mathrm{H}$ TOtal Correlation SpectroscopY (TOCSY), ${ }^{1} \mathrm{H}-{ }^{1} \mathrm{H}$ COrrelation 863 SpectroscopY (COSY), ${ }^{1} \mathrm{H}-{ }^{13} \mathrm{C}$ Hetero-nuclear Single Quantum Coherence (HSQC) and ${ }^{1} \mathrm{H}_{-}{ }^{13} \mathrm{C}$ Hetero864 nuclear Multiple-Bond Correlation (HMBC) spectroscopy were performed. Finally, when possible 865 metabolites were confirmed by in situ spiking experiments using authentic chemical standards.

866 Relative concentrations of identified metabolites from ${ }^{1} \mathrm{H}-\mathrm{NMR}$ datasets were calculated from 867 intensity measurements of a representative spectral peak of the metabolite, ensuring no overlap 868 with signals from other metabolites.

869 Euler diagram of metabolites

870 A Euler diagram of identified metabolites from Serum, Urine and Faecal biofluids associated with 871 Bariatric Surgery, Weight / BMI, T2D and Diet (pFDR <0.05) was generated using Eulerr in R (version $8726.1 .1)^{102}$.

\section{DIABLO integration of omics datasets}

874 To probe relationships between data sets we used Data Integration Analysis for Biomarker discovery 875 using Latent cOmponents (DIABLO) ${ }^{103}$, implemented through the mixOmics ${ }^{104}$ package in R. DIABLO 876 extends sparse generalized canonical correlation analysis (sGCCA) ${ }^{105}$ to a classification framework. 877 Resulting in a multi-omics integrative method that simultaneously identifies key variables correlated 878 across different data types while discriminating between phenotypic groups.

879 Normalised datasets (gut microbiota species taxa, gut microbiota KEGG pathways (Levels 2-3) and 880 quantified metabolites from urine, serum and faecal biofluids) were used with a full weighted design 881 matrix where correlation was 0.1 between data matrices and 1 for the $Y$ outcome, to result in a 882 correlated and discriminant molecular signature ${ }^{104}$. To account for the repeated measures (pre/post 883 procedure) experimental design, multilevel DIABLO (mDIABLO) models were constructed using 884 within-subject variation matrices for each omics dataset ${ }^{103,106,107}$. Classification performance was 885 assessed using the balanced error rate (BER) from cross-validation of samples, with $B E R=0.5 \times$ (false 886 positive rate + false negative rate). BER scores range from 0-1, with a perfect classification model 887 scoring 0 , a random predictor 0.5 and a model with systematically incorrect predictions 1.

888 Gut Microbiota-Metabolome associations

889 Spearman's correlations between BMI, HbA1c and Microbiota-Metabolome datasets were generated 890 in MATLAB. Corrected $p$ values (pFDR) were used to select significant correlations. Significant 
891 (pFDR<0.01) first order correlations to $\mathrm{BMI}$ and $\mathrm{HbA1c}$ and cross-correlations between these 892 variables were displayed using Cytoscape 3.8.0. ${ }^{108}$. Correlations between gut microbiota, metabolite 893 and dietary datasets were displayed using ComplexHeatmap in $\mathrm{R}^{109}$. Correlations with a pFDR value $894<0.05$ are displayed, correlations with a pFDR $<0.01$ are highlighted. Hierarchical clustering of 895 correlations was performed using Euclidean distances. 


\section{References}

897 1. Qin J, et al. A metagenome-wide association study of gut microbiota in type 2 diabetes. Nature 490, 55-60 (2012).

898
2. Ley RE, Turnbaugh PJ, Klein S, Gordon JI. Microbial ecology: human gut microbes associated with obesity. Nature 444, 1022-1023 (2006).

3. Ridaura VK, et al. Gut microbiota from twins discordant for obesity modulate metabolism in mice. Science 341, 1241214 (2013).

4. Vrieze A, et al. Transfer of intestinal microbiota from lean donors increases insulin sensitivity in individuals with metabolic syndrome. Gastroenterology 143, 913-916 e917 (2012).

5. Heintz-Buschart A, Wilmes P. Human Gut Microbiome: Function Matters. Trends Microbio/ 26, 563-574 (2018).

6. Adams TD, et al. Health benefits of gastric bypass surgery after 6 years. JAMA : the journal of the American Medical Association 308, 1122-1131 (2012).

7. Schauer PR, et al. Bariatric Surgery versus Intensive Medical Therapy for Diabetes - 5-Year Outcomes. The New England journal of medicine 376, 641-651 (2017).

8. Cummings BP, et al. Vertical sleeve gastrectomy improves glucose and lipid metabolism and delays diabetes onset in UCD-T2DM rats. Endocrinology 153, 3620-3632 (2012).

9. Liou AP, Paziuk M, Luevano JM, Jr., Machineni S, Turnbaugh PJ, Kaplan LM. Conserved shifts in the gut microbiota due to gastric bypass reduce host weight and adiposity. Science translational medicine 5, 178ra141 (2013).

10. Liu R, et al. Gut microbiome and serum metabolome alterations in obesity and after weight-loss intervention. Nature medicine 23, 859-868 (2017).

11. Aron-Wisnewsky J, et al. Major microbiota dysbiosis in severe obesity: fate after bariatric surgery. Gut 68, 70-82 (2019).

12. Turnbaugh PJ, et al. A core gut microbiome in obese and lean twins. Nature 457, 480-484 (2009).

13. Chiuve SE, et al. Alternative dietary indices both strongly predict risk of chronic disease. The Journal of nutrition 142, 1009-1018 (2012).

14. Perez-Pevida B, Escalada J, Miras AD, Fruhbeck G. Mechanisms Underlying Type 2 Diabetes Remission After Metabolic Surgery. Frontiers in endocrinology 10, 641 (2019).

15. Stenberg E, Thorell A. Insulin resistance in bariatric surgery. Current opinion in clinical nutrition and metabolic care 23, 255-261 (2020).

16. Perez-Arana GM, et al. Sleeve Gastrectomy and Roux-En-Y Gastric Bypass. Two Sculptors of the Pancreatic Islet. J Clin Med 10, (2021). 
17. Patti ME, et al. Heterogeneity of proliferative markers in pancreatic beta-cells of patients with severe hypoglycemia following Roux-en-Y gastric bypass. Acta Diabetol 54, 737-747 (2017).

944

945

946

947

948

949

950

951

952

953

954

955

956

957

958

959

960

961

962

963

964

965

966

967

968

969

970

971

972

973

974

975

976

977

978

979

980

981

982

983

984

985

986

987

988

18. Miras AD, le Roux CW. Mechanisms underlying weight loss after bariatric surgery. Nature reviews Gastroenterology \& hepatology 10, 575-584 (2013).

19. Molinaro A, Wahlstrom A, Marschall HU. Role of Bile Acids in Metabolic Control. Trends Endocrinol Metab 29, 31-41 (2018).

20. Penney NC, Kinross J, Newton RC, Purkayastha S. The role of bile acids in reducing the metabolic complications of obesity after bariatric surgery: a systematic review. International journal of obesity 39, 1565-1574 (2015).

21. Larraufie P, et al. Important Role of the GLP-1 Axis for Glucose Homeostasis after Bariatric Surgery. Cell Rep 26, 1399-1408 e1396 (2019).

22. Frezza EE, Chiriva-Internati M, Wachtel MS. Analysis of the results of sleeve gastrectomy for morbid obesity and the role of ghrelin. Surgery today $38,481-483$ (2008).

23. Jin ZL, Liu W. Progress in treatment of type 2 diabetes by bariatric surgery. World J Diabetes 12, 1187-1199 (2021).

24. Akalestou E, Miras AD, Rutter GA, le Roux CW. Mechanisms of weight loss after obesity surgery. Endocrine reviews, (2021).

25. Barberio MD, et al. Type 2 Diabetes Modifies Skeletal Muscle Gene Expression Response to Gastric Bypass Surgery. Frontiers in endocrinology 12, 728593 (2021).

26. McTigue KM, et al. Comparing the 5-Year Diabetes Outcomes of Sleeve Gastrectomy and Gastric Bypass: The National Patient-Centered Clinical Research Network (PCORNet) Bariatric Study. JAMA surgery 155, e200087 (2020).

27. Zhou W, et al. Longitudinal multi-omics of host-microbe dynamics in prediabetes. Nature 569, 663-671 (2019).

28. Forslund $\mathrm{K}$, et al. Disentangling type 2 diabetes and metformin treatment signatures in the human gut microbiota. Nature 528, 262-266 (2015).

29. Wu $\mathrm{H}$, et al. Metformin alters the gut microbiome of individuals with treatment-naive type 2 diabetes, contributing to the therapeutic effects of the drug. Nature medicine 23, 850-858 (2017).

30. Wang TJ, et al. 2-Aminoadipic acid is a biomarker for diabetes risk. The Journal of clinical investigation 123, 43094317 (2013).

31. Razquin C, et al. Lysine pathway metabolites and the risk of type 2 diabetes and cardiovascular disease in the PREDIMED study: results from two case-cohort studies. Cardiovascular diabetology 18, 151 (2019).

32. Xu WY, et al. 2-Aminoadipic acid protects against obesity and diabetes. J Endocrinol 243, 111-123 (2019). 
33. Thorpe SR, Baynes JW. Role of the Maillard reaction in diabetes mellitus and diseases of aging. Drugs Aging $\mathbf{9}$, 69-77 (1996).

34. Connick JH, Stone TW. The role of kynurenines in diabetes mellitus. Med Hypotheses 18, 371-376 (1985).

35. Hirayama A, et al. Metabolic profiling reveals new serum biomarkers for differentiating diabetic nephropathy. Analytical and bioanalytical chemistry 404, 3101-3109 (2012).

36. Liang X, Kaya A, Zhang Y, Le DT, Hua D, Gladyshev VN. Characterization of methionine oxidation and methionine sulfoxide reduction using methionine-rich cysteine-free proteins. BMC Biochem 13, 21 (2012).

37. le Roux CW, et al. Gut hormone profiles following bariatric surgery favor an anorectic state, facilitate weight loss, and improve metabolic parameters. Annals of surgery 243, 108-114 (2006).

38. Scholtz $\mathrm{S}$, et al. Obese patients after gastric bypass surgery have lower brain-hedonic responses to food than after gastric banding. Gut 63, 891-902 (2014).

39. Ahmed K, Penney N, Darzi A, Purkayastha S. Taste Changes after Bariatric Surgery: a Systematic Review. Obesity surgery 28, 3321-3332 (2018).

40. Rennie KL, Coward A, Jebb SA. Estimating under-reporting of energy intake in dietary surveys using an individualised method. The British journal of nutrition 97, 1169-1176 (2007).

41. Donaldson GP, Lee SM, Mazmanian SK. Gut biogeography of the bacterial microbiota. Nature reviews Microbiology 14, 20-32 (2016).

42. Zoetendal EG, et al. The human small intestinal microbiota is driven by rapid uptake and conversion of simple carbohydrates. ISME J 6, 1415-1426 (2012).

43. Suzuki TA, Nachman MW. Spatial Heterogeneity of Gut Microbial Composition along the Gastrointestinal Tract in Natural Populations of House Mice. PloS one 11, e0163720 (2016).

44. Miquel S, et al. Ecology and metabolism of the beneficial intestinal commensal bacterium Faecalibacterium prausnitzii. Gut microbes 5, 146-151 (2014).

45. Ley RE, Backhed F, Turnbaugh P, Lozupone CA, Knight RD, Gordon Jl. Obesity alters gut microbial ecology. Proceedings of the National Academy of Sciences of the United States of America 102, 11070-11075 (2005).

46. Duncan $\mathrm{SH}$, et al. Human colonic microbiota associated with diet, obesity and weight loss. International journal of obesity 32, 1720-1724 (2008).

47. Schwiertz A, et al. Microbiota and SCFA in lean and overweight healthy subjects. Obesity 18, 190-195 (2010).

48. Sze MA, Schloss PD. Looking for a Signal in the Noise: Revisiting Obesity and the Microbiome. mBio 7, (2016).

49. Elliott $\mathrm{P}$, et al. Urinary metabolic signatures of human adiposity. Science translational medicine 7, $285 \mathrm{ra262}$ (2015). 
037 038

039

040

041

042

043

044

045

046

047

048

049

050

051

052

053

054

055

056

057

058

059

060

061

062

063

064

065

066

067

068

069

070

071

072

073

074

075

076

077

078

079

080

081

082

083

084
50. Li JV, et al. Metabolic surgery profoundly influences gut microbial-host metabolic cross-talk. Gut 60, 1214-1223 (2011).

51. Pallister $\mathrm{T}$, et al. Hippurate as a metabolomic marker of gut microbiome diversity: Modulation by diet and relationship to metabolic syndrome. Sci Rep 7, 13670 (2017).

52. Tai ES, et al. Insulin resistance is associated with a metabolic profile of altered protein metabolism in Chinese and Asian-Indian men. Diabetologia 53, 757-767 (2010).

53. Wang TJ, et al. Metabolite profiles and the risk of developing diabetes. Nature medicine 17, 448-453 (2011).

54. Soeters PB, Fischer JE. Insulin, glucagon, aminoacid imbalance, and hepatic encephalopathy. Lancet 2, 880-882 (1976).

55. Libert DM, Nowacki AS, Natowicz MR. Metabolomic analysis of obesity, metabolic syndrome, and type 2 diabetes: amino acid and acylcarnitine levels change along a spectrum of metabolic wellness. PeerJ 6, e5410 (2018).

56. White PJ, Newgard CB. Branched-chain amino acids in disease. Science 363, 582-583 (2019).

57. Tremblay F, et al. Identification of IRS-1 Ser-1101 as a target of S6K1 in nutrient- and obesity-induced insulin resistance. Proceedings of the National Academy of Sciences of the United States of America 104, 14056-14061 (2007).

58. Mardinoglu A, et al. The gut microbiota modulates host amino acid and glutathione metabolism in mice. Molecular systems biology 11, 834 (2015).

59. Pedersen HK, et al. Human gut microbes impact host serum metabolome and insulin sensitivity. Nature 535, 376-381 (2016).

60. Dai ZL, Wu G, Zhu WY. Amino acid metabolism in intestinal bacteria: links between gut ecology and host health. Front Biosci (Landmark Ed) 16, 1768-1786 (2011).

61. Hoyles L, et al. Metabolic retroconversion of trimethylamine N-oxide and the gut microbiota. Microbiome 6, 73 (2018).

62. Koeth RA, et al. Intestinal microbiota metabolism of L-carnitine, a nutrient in red meat, promotes atherosclerosis. Nature medicine 19, 576-585 (2013).

63. Mueller DM, et al. Plasma levels of trimethylamine-N-oxide are confounded by impaired kidney function and poor metabolic control. Atherosclerosis 243, 638-644 (2015).

64. Barton $\mathrm{W}$, et al. The microbiome of professional athletes differs from that of more sedentary subjects in composition and particularly at the functional metabolic level. Gut 67, 625-633 (2018).

65. Holmes $\mathrm{E}$, et al. Human metabolic phenotype diversity and its association with diet and blood pressure. Nature 453, 396-400 (2008). 
66. Dumas ME, et al. Microbial-Host Co-metabolites Are Prodromal Markers Predicting Phenotypic Heterogeneity in Behavior, Obesity, and Impaired Glucose Tolerance. Cell Rep 20, 136-148 (2017).

67. Hardison WG, Grundy SM. Effect of bile acid conjugation pattern on bile acid metabolism in normal humans. Gastroenterology 84, 617-620 (1983).

68. Swann JR, et al. Systemic gut microbial modulation of bile acid metabolism in host tissue compartments. Proceedings of the National Academy of Sciences of the United States of America 108 Suppl 1, 4523-4530 (2011).

69. Zheng $X$, et al. Hyocholic acid species as novel biomarkers for metabolic disorders. Nature communications $\mathbf{1 2}$, 1487 (2021).

70. Zheng $\mathrm{X}$, et al. Hyocholic acid species improve glucose homeostasis through a distinct TGR5 and FXR signaling mechanism. Cell metabolism 33, 791-803 e797 (2021).

71. Perry RJ, et al. Acetate mediates a microbiome-brain-beta-cell axis to promote metabolic syndrome. Nature 534, 213-217 (2016).

72. Lin HV, et al. Butyrate and propionate protect against diet-induced obesity and regulate gut hormones via free fatty acid receptor 3-independent mechanisms. PloS one 7, e35240 (2012).

73. Windey K, De Preter V, Verbeke K. Relevance of protein fermentation to gut health. Mol Nutr Food Res 56, 184196 (2012).

74. Hughes R, Magee EA, Bingham S. Protein degradation in the large intestine: relevance to colorectal cancer. Curr Issues Intest Microbiol 1, 51-58 (2000).

75. Li JV, et al. Experimental bariatric surgery in rats generates a cytotoxic chemical environment in the gut contents. Frontiers in microbiology 2, 183 (2011).

76. Sun $\mathrm{CY}$, Hsu HH, Wu MS. p-Cresol sulfate and indoxyl sulfate induce similar cellular inflammatory gene expressions in cultured proximal renal tubular cells. Nephrol Dial Transplant 28, 70-78 (2013).

77. Gratton J, et al. Optimized Sample Handling Strategy for Metabolic Profiling of Human Feces. Analytical chemistry 88, 4661-4668 (2016).

78. Dona AC, et al. Precision high-throughput proton NMR spectroscopy of human urine, serum, and plasma for large-scale metabolic phenotyping. Analytical chemistry 86, 9887-9894 (2014).

79. Veselkov KA, et al. Optimized preprocessing of ultra-performance liquid chromatography/mass spectrometry urinary metabolic profiles for improved information recovery. Analytical chemistry 83, 5864-5872 (2011).

80. Sarafian $\mathrm{MH}$, et al. Bile acid profiling and quantification in biofluids using ultra-performance liquid chromatography tandem mass spectrometry. Analytical chemistry 87, 9662-9670 (2015).

81. Moreau NM, et al. Simultaneous measurement of plasma concentrations and 13C-enrichment of short-chain fatty acids, lactic acid and ketone bodies by gas chromatography coupled to mass spectrometry. Journal of chromatography B, Analytical technologies in the biomedical and life sciences 784, 395-403 (2003). 
82. Siskos AP, et al. Interlaboratory Reproducibility of a Targeted Metabolomics Platform for Analysis of Human Serum and Plasma. Analytical chemistry 89, 656-665 (2017).

83. $\mathrm{Xu} \mathrm{H}$, et al. FastUniq: a fast de novo duplicates removal tool for paired short reads. PloS one 7, e52249 (2012).

84. Hoyles L, et al. Molecular phenomics and metagenomics of hepatic steatosis in non-diabetic obese women. Nature medicine 24, 1070-1080 (2018).

85. Li H. Aligning sequence reads, clone sequences and assembly contigs with BWA-MEM. arXiv, (2013).

86. Truong DT, et al. MetaPhIAn2 for enhanced metagenomic taxonomic profiling. Nat Methods 12, 902-903 (2015).

87. Segata N, Waldron L, Ballarini A, Narasimhan V, Jousson O, Huttenhower C. Metagenomic microbial community profiling using unique clade-specific marker genes. Nat Methods $\mathbf{9}, 811-814$ (2012).

88. Nurk S, Meleshko D, Korobeynikov A, Pevzner PA. metaSPAdes: a new versatile metagenomic assembler. Genome Res 27, 824-834 (2017).

89. Zhu W, Lomsadze A, Borodovsky M. Ab initio gene identification in metagenomic sequences. Nucleic Acids Res 38, e132 (2010).

90. Besemer J, Borodovsky M. Heuristic approach to deriving models for gene finding. Nucleic Acids Res 27, 39113920 (1999).

91. Edgar RC. Search and clustering orders of magnitude faster than BLAST. Bioinformatics 26, 2460-2461 (2010).

92. Le Chatelier E, et al. Richness of human gut microbiome correlates with metabolic markers. Nature 500, 541546 (2013).

93. Huerta-Cepas J, et al. Fast Genome-Wide Functional Annotation through Orthology Assignment by eggNOGMapper. Mol Biol Evol 34, 2115-2122 (2017).

94. Qin N, et al. Alterations of the human gut microbiome in liver cirrhosis. Nature 513, 59-64 (2014).

95. Warrack BM, et al. Normalization strategies for metabonomic analysis of urine samples. Journal of chromatography B, Analytical technologies in the biomedical and life sciences 877, 547-552 (2009).

96. Asnicar F, Weingart G, Tickle TL, Huttenhower C, Segata N. Compact graphical representation of phylogenetic data and metadata with GraPhIAn. PeerJ 3, e1029 (2015).

97. Posma JM, et al. Optimized Phenotypic Biomarker Discovery and Confounder Elimination via Covariate-Adjusted Projection to Latent Structures from Metabolic Spectroscopy Data. Journal of proteome research 17, 1586-1595 (2018).

98. Garcia-Perez I, et al. Objective assessment of dietary patterns by use of metabolic phenotyping: a randomised, controlled, crossover trial. The lancet Diabetes \& endocrinology 5, 184-195 (2017). 
99. Oksanen J, et al. vegan: Community Ecology Package. R package version 2.5-6, (2019).

100. Posma JM, et al. Subset optimization by reference matching (STORM): an optimized statistical approach for recovery of metabolic biomarker structural information from $1 \mathrm{H}$ NMR spectra of biofluids. Analytical chemistry 84, 10694-10701 (2012).

101. Cloarec 0 , et al. Statistical total correlation spectroscopy: an exploratory approach for latent biomarker identification from metabolic 1 H NMR data sets. Analytical chemistry 77, 1282-1289 (2005).

102. Larsson J. eulerr: Area-Proportional Euler and Venn Diagrams with Ellipses. R package version 6.1.1. (2021).

103. Singh A, et al. DIABLO: an integrative approach for identifying key molecular drivers from multi-omics assays. Bioinformatics 35, 3055-3062 (2019).

104. Rohart F, Gautier B, Singh A, Le Cao KA. mixOmics: An R package for 'omics feature selection and multiple data integration. PLoS Comput Biol 13, e1005752 (2017).

105. Tenenhaus A, Philippe C, Guillemot V, Le Cao KA, Grill J, Frouin V. Variable selection for generalized canonical correlation analysis. Biostatistics 15, 569-583 (2014).

106. Liquet B, Le Cao KA, Hocini H, Thiebaut R. A novel approach for biomarker selection and the integration of repeated measures experiments from two assays. BMC bioinformatics 13, 325 (2012).

107. Westerhuis JA, van Velzen EJ, Hoefsloot HC, Smilde AK. Multivariate paired data analysis: multilevel PLSDA versus OPLSDA. Metabolomics 6, 119-128 (2010).

108. Shannon P, et al. Cytoscape: a software environment for integrated models of biomolecular interaction networks. Genome Res 13, 2498-2504 (2003).

109. Gu Z, Eils R, Schlesner M. Complex heatmaps reveal patterns and correlations in multidimensional genomic data. Bioinformatics 32, 2847-2849 (2016). 


\section{Acknowledgments:}

215 This research was funded by the Diabetes Research and Wellness Foundation through the 216 Sutherland-Earl Clinical Research Fellowship 2015 awarded to NP.

217 The research was supported by the National Institute for Health Research (NIHR) Imperial Biomedical 218 Research Centre (BRC) and Clinical Research Network (CRN). The views expressed are those of the 219 authors and not necessarily those of the NHS, NIHR or UK Department of Health and Social Care.

220 We are grateful to the Imperial Weight Centre for facilitating this research.

221 This work used the computing resources of the UK MEDical BIOinformatics partnership - aggregation, 222 integration, visualisation and analysis of large, complex data (UK Med-Bio) - which was supported by 223 the Medical Research Council (grant number MR/L01632X/1).

224 IGP is supported by a NIHR Career Development Research Fellowship (NIHR-CDF-2017-10-032). JMP 225 is supported by a Rutherford Fund Fellowship at Health Data Research (HDR) UK (MR/S004033/1). 226 GF is supported by an NIHR Senior Investigator award. LH was in receipt of an MRC Intermediate 227 Research Fellowship in Data Science (MR/L01632X/1, UK MED-BIO). EH is supported by the 228 Department of Jobs, Tourism, Science and Innovation, Government of Western Australian through 229 the Premier's Science Fellowship Program and by the Australian Research Council Laureate 230 Fellowship Scheme.

\section{Author contributions:}

233 Conceptualisation: NP, EH, JM, AD. Funding acquisition: NP, SP. Data curation: NP, DY, BG. 234 Microbiome Investigation: NP, LH, JM. Metabolite Investigation: NP, IGP, EH. Microbiome Analysis: 235 LH, NP. Metabolite Analysis: NP, IGP, EH. Dietary Analysis: AK, NP, GF. Software: JMP, GF. Supervision: $236 \mathrm{EH}, \mathrm{SP}, \mathrm{JM}, \mathrm{GF}, \mathrm{AD}$. Resources: EH, SP, JM, GF, AD. Writing - Original Draft: NP. Writing - Review \& 237 Editing: All authors.

239 Declaration of interests:

240 The authors declare no competing interests. 


\section{Supplementary Files}

This is a list of supplementary files associated with this preprint. Click to download.

- SupplementallnformationFINAL.pdf 